\title{
WEAK STABILITY BOUNDARY AND INVARIANT MANIFOLDS
}

\author{
EDWARD BELBRUNO*, MARIAN GIDEA ${ }^{\dagger}$, AND FRANCESCO TOPPUTO $\ddagger$
}

\begin{abstract}
The concept of weak stability boundary has been successfully used in the design of several fuel efficient space missions. In this paper we give a rigorous definition of the weak stability boundary in the context of the planar circular restricted three-body problem, and we provide a geometric argument for the fact that, for some energy range, the points in the weak stability boundary of the small primary are the points with zero radial velocity that lie on the stable manifolds of the Lyapunov orbits about the libration points $L_{1}$ and $L_{2}$, provided that these manifolds satisfy some topological conditions. The geometric method is based on the property of the invariant manifolds of Lyapunov orbits being separatrices of the energy manifold. We support our geometric argument with numerical experiments.
\end{abstract}

Key words. Weak Stability Boundary, Lyapunov Orbits, Invariant Manifolds

AMS subject classifications. 70F $15,70 \mathrm{~F} 07,37 \mathrm{D} 10$

1. Introduction. The notion of weak stability boundary (WSB) was first introduced heuristically by Belbruno in 1987 for designing fuel efficient space missions and was proven to be useful in applications $[2,3,4,12,13,6,45,33,14,35,22,7,39,38,44]$. The WSB can be used to construct low energy transfers to the Moon, requiring little or no fuel for capture into lunar orbit. The first application for an operational spacecraft occurred in 1991 with the rescue of the Japanese mission Hiten. WSB was also applied in ESA's spacecraft SMART-1 in 2004 (see [40]). The WSB technique will be applied again in ESA's mission BepiColombo to explore planet Mercury in 2013 (see [25]), and in some upcoming NASA missions.

A different methodology of designing fuel efficient trajectories, based on hyperbolic invariant manifolds, was proposed in [1, 26, 28, 29, 30,31], and was successfully applied in several space missions (see also [18, 19, 20, 21]). In some of these works it has been suggested that the hyperbolic invariant manifold method can be used to explain the trajectories obtained through the WSB method. Supporting this assertion, García and Gómez present in [22] numerical explorations that suggests that, for some range of energies, the WSB is contained in the closure of the union of the stable manifolds of the periodic orbits about two of the equilibrium points of the planar circular restricted three-body problem.

In this paper we use the separatrix property of the invariant manifolds of the periodic orbits about the equilibrium points to argue that, under some conditions, the points on the stable manifolds are WSB points. We support our geometrical argument with numerical experiments. Our result, corroborated with the result in [22], demonstrates, by double inclusion, that, for some range of energies, the WSB coincides with the set of points on the stable manifolds with zero radial velocity and negative Kepler energy relative to the small primary.

In Section 2 we give some background on the planar circular restricted three-body problem. In Section 3 we define the WSB as follows: in the context of the planar circular restricted three-body problem, for each radial segment emanating from the

\footnotetext{
*Department of Astrophysical Sciences, Princeton University, Peyton Hall, Ivy Lane, Princeton, NJ 08544, USA (belbruno@princeton.edu).

$\dagger$ Department of Mathematics, Northeastern Illinois University 5500 N. St. Louis Avenue, Chicago, IL 60625, USA (mgidea@neiu.edu)

$\ddagger$ Aerospace Engineering Department, Politecnico di Milano Via La Masa, 34, 20156, Milan, Italy (topputo@aero.polimi.it)
} 
small primary, we consider trajectories that leave that segment at the periapsis of an osculating ellipse whose semi-major axis is a part of the radial segment; a trajectory is called $n$-stable if it makes $n$ full turns around the small primary without going about the large primary and it has negative Kepler energy when it returns to the radial segment; otherwise the trajectory is called $n$-unstable; the points that make the transition from the $n$-stable regime to the $n$-unstable one are by definition the points of the WSB of index $n$. We make precise what means a full turn about a primary through the measurement of the net change in the angle swept by the position vector. We also require that the $n$ intersections of the trajectory with the radial line are transverse. In this way, the $n$-stability condition is an open condition and the set of the $n$-stable points on the radial segment is a countable union of open intervals. Thus, the WSB consists in the union of endpoints of such intervals, resembling a Cantor set (in agreement with [22]). In Section 4 we give a numerical and geometric argument for the fact that the points on the stable manifolds of the periodic orbits around the libration points $L_{1}$ and $L_{2}$, with the property that they have zero radial velocity and negative Kepler energy relative to the small primary, are contained in the WSB, for some range of energies. The key idea for our argument is the separatrix property of these invariant manifolds, meaning that these manifolds separate two types of motions: transfer orbits between the primaries, and non-transfer orbits. Section 5 exposes the main conclusions of this paper: the WSB theory overlaps with the invariant manifold theory for a significant range of energies.

It is important to note that the WSB is not an invariant object for the dynamics. It is therefore somewhat surprising that the WSB is related to hyperbolic invariant manifolds. The remarkable feature of the weak stability boundary is that the local behavior near the small primary and hence far from the equilibrium points is sufficient to assess whether the trajectory lies on the stable manifold of a periodic orbit near an equilibrium point.

We remark that the definition of the WSB does not rely on the existence of the hyperbolic invariant manifolds. That is, one can still define and apply the properties of the WSB in models where the hyperbolic invariant manifolds are no longer well defined or they are not separatrices. Some examples include the elliptic restricted three-body problem and the bicircular restricted four body problem, for which WSB sets were computed in [38]. These problems are described by non-autonomous Hamiltonian systems where the notions of stable and unstable manifolds are not well-defined in the phase space ${ }^{1}$. It seems possible that the WSB may turn out to provide a good substitute for the hyperbolic invariant manifolds in such models (see [23]).

\section{Background.}

2.1. Planar Circular Restricted Three-Body Problem. The model that we use to describe the motion of an infinitesimal particle relative to the Earth-Moon system is the planar circular restricted three-body problem (PCRTBP). In this model, relative to an inertial frame, two primary bodies $P_{1}, P_{2}$ of masses $m_{1}>m_{2}>0$, respectively, move under mutual gravity on circular orbits about their common center of mass. The third body $P_{3}$, assumed of infinitesimal mass, moves under the gravity of

\footnotetext{
${ }^{1}$ One can transform a non-autonomous Hamiltonian system into autonomous one by adding an extra variable symplectically conjugated with time. Then one can compute invariant manifolds for the extended system. However, these invariant manifolds contain a dependency on time, so they do not separate transfer orbits from non-transfer orbits. If one considers a time-discretization of the system by a Poincaré map, one obtains time-independent invariant manifolds, but these manifolds again do not separate transfer orbits from non-transfer orbits.
} 
the primaries in the same plane as them. The motion of the primaries is not affected by the motion of the infinitesimal mass. In our case, $P_{3}$ represents a spacecraft, and $P_{1}, P_{2}$ represent the Earth and the Moon, respectively. Let the mass ratio of the small body vs. total mass be $\mu=m_{2} /\left(m_{1}+m_{2}\right)$. In the following we will consider $\mu=0.0121506683$.

The motion of the infinitesimal mass, relative to a co-rotating coordinate system $(x, y)$ with the origin at the center of mass of the two bodies, and in normalized units of distance, mass and time, is described by the following equations (following [43]):

$$
\ddot{x}-2 \dot{y}=\frac{\partial \Omega}{\partial x}, \quad \ddot{y}+2 \dot{x}=\frac{\partial \Omega}{\partial y},
$$

where the effective potential $\Omega$ is given by

$$
\Omega(x, y)=\frac{1}{2}\left(x^{2}+y^{2}\right)+\frac{1-\mu}{r_{1}}+\frac{\mu}{r_{2}}+\frac{1}{2} \mu(1-\mu),
$$

with $r_{1}=\left((x+\mu)^{2}+y^{2}\right)^{1 / 2}, r_{2}=\left((x+\mu-1)^{2}+y^{2}\right)^{1 / 2}$ as $P_{1}, P_{2}$ are located at $(-\mu, 0),(1-\mu, 0)$, respectively.

The problem has the following symmetry

$$
(x, y, \dot{x}, \dot{y}, t) \longrightarrow(x,-y,-\dot{x}, \dot{y},-t),
$$

meaning that each solution of (2.1) is either symmetric or has a symmetric counterpart.

The motion described by (2.1) has five equilibrium points $L_{k}, k=1,2, \ldots, 5$, known as the Euler-Lagrange libration points. Three of these, $L_{1}, L_{2}, L_{3}$, lie along the $x$ axis, at the approximate coordinates $x_{1}=0.8369147188, x_{2}=1.1556824834, x_{3}=$ -1.0050626802 , respectively. In our notation $L_{1}$ lies between the Earth and Moon, and $L_{2}$ lies outside the Moon. The other two points, $L_{4}, L_{5}$, lie at the vertices of two equilateral triangles with common base extending from $P_{1}$ to $P_{2}$.

The system of differential equations (2.1) admits an integral of motion, the Jacobi integral,

$$
J(x, y, \dot{x}, \dot{y})=2 \Omega(x, y)-\left(\dot{x}^{2}+\dot{y}^{2}\right) .
$$

The projection of an energy manifold

$$
\mathcal{J}(C)=\left\{(x, y, \dot{x}, \dot{y}) \in \mathbb{R}^{4} \mid J(x, y, \dot{x}, \dot{y})=C\right\},
$$

onto the configuration space $(x, y)$ is called a Hill's region. The motion of $P_{3}$ is always confined to the Hill's region of the corresponding Jacobi energy $C$. The boundary of a Hill's region is a zero-velocity curve. The Hill regions vary with the Jacobi energy $C$ (see [43]). The Jacobi constants corresponding to the libration points are $C_{1} \approx 3.2003449098, C_{2} \approx 3.1841641431, C_{3} \approx 3.0241502628$, and $C_{4}=C_{5}=3$.

2.2. Equations of motion in polar coordinates. The equations of motion (2.1) can be written in polar coordinates $(r, \theta)$ relative to $P_{2}$, where $r$ is the distance from $P_{2}$ to $P_{3}$ and $\theta$ is the angle between the axis $P_{1} P_{2}$ and $P_{2} P_{3}$ measured counterclockwise. See [44].

If the motion of $P_{3}$ starts at the periapsis $(\dot{r}=0)$ of an osculating ellipse around the Moon of semi-major axis $a$ and eccentricity $e \in[0,1)$, and has initial velocity $v$ with respect to the sidereal reference frame, then we have

$$
r=a(1-e), \quad v=\sqrt{\frac{(1+e) \mu}{r}} .
$$


The Jacobi integral of the motion takes the form $(\dot{r}=0)$ :

$$
\begin{aligned}
J(r, v, \theta)= & (1-\mu)\left[1+2 r \cos \theta+\frac{2}{\sqrt{r^{2}+2 r \cos \theta+1}}\right]+\frac{2 \mu}{r}+2 v r-v^{2}, \text { or } \\
J(r, e, \theta)= & (1-\mu)\left[1+2 r \cos \theta+\frac{2}{\sqrt{r^{2}+2 r \cos \theta+1}}\right]+\frac{2 \mu}{r} \\
& +2 \sqrt{\frac{(1+e) \mu}{r}} r-\frac{(1+e) \mu}{r} .
\end{aligned}
$$

In the definition of the WSB, we will need to refer to the Kepler energy $\mathrm{H}_{2}$ of $P_{3}$ with respect to the the primary $P_{2}$. This is given by

$$
H_{2}=\frac{1}{2} v^{2}-\frac{\mu}{r} .
$$

From (2.6) it follows

$$
H_{2}=\frac{(e-1) \mu}{2 r} .
$$

2.3. Invariant Manifolds of Lyapunov Orbits. The collinear libration points $L_{i}, i \in\{1,2,3\}$, are of center-saddle type. For some range of energies $C$, there exists a one-parameter family of periodic orbits $\left\{\gamma_{i}(C)\right\}_{C}$, also called Lyapunov orbits, emanating from the libration point $L_{i}$. As the energy $C$ is decreased, the Lyapunov family of $L_{i}$ approach the closest primary and collision occurs. When the energy is further decreased, the Lyapunov family continues to periodic orbits around the closest primary; such orbits can describe multiple loops about the primary, which can eventually shrink or coalesce into one loop. See $[43,8,16]$.

For fixed $C$ in some appropriate energy range, each periodic orbit $\gamma_{i}$ possesses stable and unstable manifolds $W^{s}\left(\gamma_{i}\right)$ and $W^{u}\left(\gamma_{i}\right)$. For some values of $C$, the manifolds experience collisions and close encounters with the primaries. To avoid ill conditioning and increase of errors during numerical integration the equations of motion have been regularized, using Levi-Civita regularization [43], when the motion of the small particle is inside a disc of radius $10^{-2}$ around either primary.

The stable and unstable manifolds are 2-dimensional manifolds locally diffeomorphic to cylinders in the 3-dimensional energy manifold, and they are separatrices of the dynamics restricted to the energy manifold (see [15]). This means that, for $C \lesssim C_{2}$, the trajectories starting inside the stable cylinder make a transfer from one lobe of the Hill's region to the other lobe or to the exterior region in forward time, while the trajectories starting inside the unstable cylinder make a transfer from one lobe of the Hill's region to the other lobe or to the exterior region in backwards time. See Fig. 2.1.

2.4. Poincaré sections. We consider the cuts made by the stable and unstable manifolds of the Lyapunov orbits about $L_{1}$ and $L_{2}$ for a fixed Jacobi energy $C$, with a varying Poincaré section through $P_{2}$. Let

$$
\mathcal{S}_{\theta_{0}}=\left\{(r, \dot{r}, \theta, \dot{\theta}) \mid \theta=\theta_{0}, \dot{\theta}>0\right\},
$$

be the Poincaré section through $P_{2}$ which makes an angle $\theta_{0}$ with $P_{1} P_{2}$. The coordinates $(r, \dot{r})$ of a point in $\mathcal{S}_{\theta_{0}}$ determines a unique trajectory through that point: the $\theta$-coordinate equals $\theta_{0}$ in this section, and the $\dot{\theta}$-coordinate can be solved uniquely 


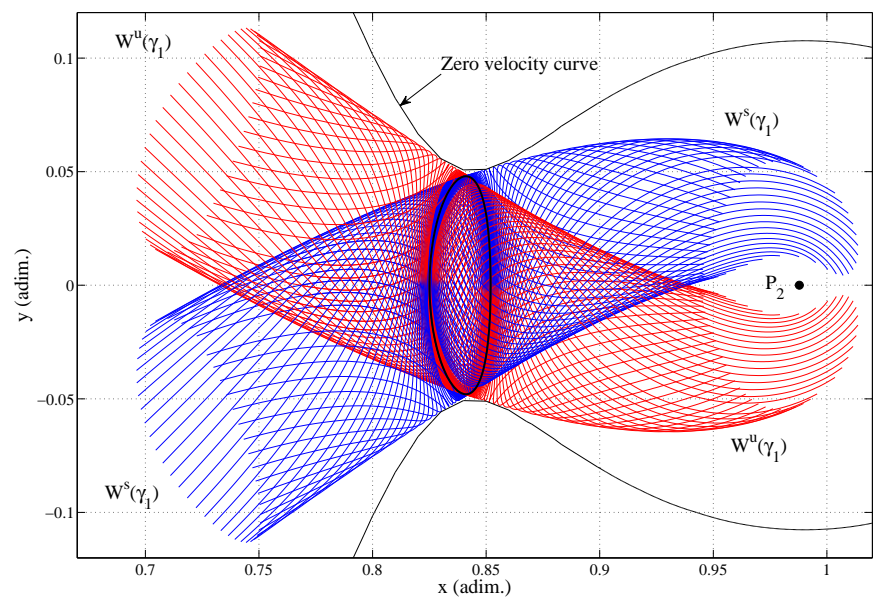

FIG. 2.1. Zero velocity curves, Lyapunov orbit $\gamma_{1}$, and two branches of the stable and unstable manifolds, $W^{s}\left(\gamma_{1}\right)$ and $W^{u}\left(\gamma_{1}\right)$, for $C=3.19$.

from the energy condition $J(r, \dot{r}, \theta, \dot{\theta})=C$, provided $\dot{\theta}>0$. Thus, each section $\mathcal{S}_{\theta_{0}}$ is plotted as an $(r, \dot{r})$-coordinate plane. The Poincaré first return map to $\mathcal{S}_{\theta_{0}}$ is denoted by $\Phi_{\theta_{0}}$.

We are interested in the successive cuts made by the invariant manifolds of $\gamma_{i}$, $i=1,2$, with $\mathcal{S}_{\theta_{0}}$, where $\gamma_{i}$ is the Lyapunov orbit about $L_{i}$ for a fixed energy level $C$. We label the cuts in a way that the label of each cut matches with the number of complete turns about $P_{2}$ made by a trajectory starting from that cut until it approaches $\gamma_{i}$ (in positive or negative time). We denote by $W_{\theta_{0}, j}^{s}\left(\gamma_{i}\right)$ the cut made by the stable manifold $W^{s}\left(\gamma_{i}\right)$ with $\mathcal{S}_{\theta_{0}}$ with the property that all the points in this cut make $j$-complete turns about $P_{2}$ before approaching $\gamma_{i}$ in forward time. Similarly, we denote by $W_{\theta_{0}, j}^{u}\left(\gamma_{i}\right)$ the cut made by the unstable manifold $W^{u}\left(\gamma_{i}\right)$ with $\mathcal{S}_{\theta_{0}}$ with the property that all the points in this cut make $j$-complete turns about $P_{2}$ before approaching $\gamma_{i}$ in backwards time. This labels can be assigned inductively in the manner described below.

Consider the branch of $W^{s}\left(\gamma_{1}\right)$ in the $P_{2}$-region, and a varying Poincaré section $\mathcal{S}_{\theta_{0}}$ rotating clockwise about $P_{2}$. Assume that $W^{s}\left(\gamma_{1}\right)$ does not collide with $P_{2}$ and turns around $P_{2}$. Let $-\theta_{1}$ and $\theta_{1}$ be the angles made by the tangent lines from $P_{2}$ to the Lyapunov orbit $\gamma_{1}$. For $-\theta_{1}<\theta_{0}<\theta_{1}$, the first cut between $W^{s}\left(\gamma_{1}\right)$ with $\mathcal{S}_{\theta_{0}}$ is not well defined, as the trajectories on the stable manifold approach asymptotically $\gamma_{1}$ and intersect $\mathcal{S}_{\theta_{0}}$ infinitely many times. If we rotate clockwise $\mathcal{S}_{\theta_{0}}$ to an angle $\theta_{1}<\theta_{0}<2 \pi-\theta_{1}$, the first cut between $W^{s}\left(\gamma_{1}\right)$ with $\mathcal{S}_{\theta_{0}}$ is well defined, but the trajectories starting from this first cut make less than 1-turn about $P_{2}$ before approaching $\gamma_{1}$. For these values of $\theta_{0}$, we denote the first cut made by $W^{s}\left(\gamma_{1}\right)$ with $\mathcal{S}_{\theta_{0}}$ by $W_{\theta_{0}, 0}^{s}\left(\gamma_{1}\right)$. The angle $\theta(t)$ swept by a trajectory starting from $W_{\theta_{0}, 0}^{s}\left(\gamma_{1}\right)$ until it approaches asymptotically $\gamma_{1}$ is less than $2 \pi$, that is, such a trajectory completes 0 -turns about $P_{2}$. When we rotate clockwise $\mathcal{S}_{\theta_{0}}$ to an angle $2 \pi-\theta_{1}<\theta_{0}<2 \pi+\theta_{1}$, the first cut of $W^{s}\left(\gamma_{1}\right)$ with $\mathcal{S}_{\theta_{0}}$ is still well defined if we restrict the trajectories off some convenient neighborhood of $\gamma_{1}$. The angle $\theta(t)$ swept by a trajectory starting 


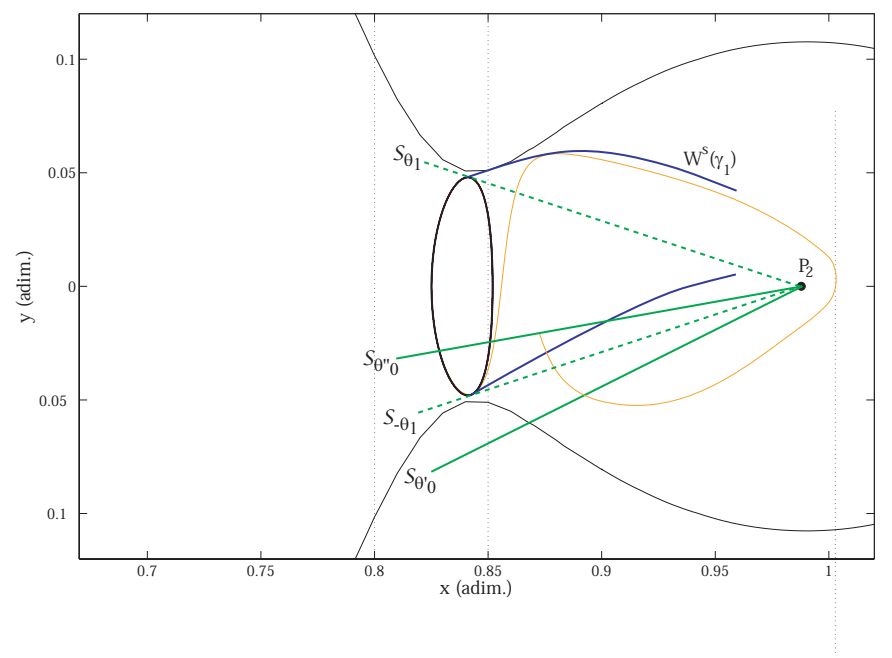

FIG. 2.2. A trajectory that completes 0 -turns if it originates from $\mathcal{S}_{\theta_{0}^{\prime}}$ with $-\theta_{1}<\theta_{0}^{\prime}<2 \pi-\theta_{1}$, and makes 1 -complete turn around $P_{2}$ if it originates from $\mathcal{S}_{\theta_{0}^{\prime \prime}}$ with $2 \pi-\theta_{1}<\theta_{0}^{\prime \prime}<4 \pi-\theta_{1}$. The angles are measured clockwise starting from $P_{2} P_{1}$.

from this first cut until it approaches asymptotically $\gamma_{1}$ is a little more than $2 \pi$, thus such a trajectory completes 1-turn about $P_{2}$, and the cut is labeled by $W_{\theta_{0}, 1}^{s}\left(\gamma_{1}\right)$. If we rotate clockwise $\mathcal{S}_{\theta_{0}}$ to an angle $2 \pi+\theta_{1}<\theta_{0}<4 \pi-\theta_{1}$, the second cut of $W^{s}\left(\gamma_{1}\right)$ with $\mathcal{S}_{\theta_{0}}$ consists of points that still make 1-turns about $P_{2}$ until they approach $\gamma_{1}$, so such a cut is denoted by $W_{\theta_{0}, 1}^{s}\left(\gamma_{1}\right)$. For $4 \pi-\theta_{1}<\theta_{0}<6 \pi-\theta_{1}$ a similar argument yields a cut with the property that the trajectories emerging from this cut completes 2-turns about $P_{2}$ until it approaches $\gamma_{1}$, so such a cut is denoted by $W_{\theta_{0}, 2}^{s}\left(\gamma_{1}\right)$. Inductively, this procedure produces a labeling $W_{\theta_{0}, j}^{s}\left(\gamma_{1}\right)$ for the cuts of $W^{s}\left(\gamma_{1}\right)$ with $\mathcal{S}_{\theta_{0}}$ with $2 j \pi-\theta_{1}<\theta_{0}<2(j+1) \pi-\theta_{1}$, where $j \geq 0$. The trajectories staring from $W_{\theta_{0}, j}^{s}\left(\gamma_{1}\right)$ complete $j$-turns about $P_{2}$ until they approach $\gamma_{1}$ in forward time. In a similar manner, one can produce a labeling $W_{\theta_{0}, j}^{u}\left(\gamma_{1}\right)$ of the cuts of $W^{u}\left(\gamma_{1}\right)$ with $\mathcal{S}_{\theta_{0}}$, such that the trajectories staring from $W_{\theta_{0}, j}^{u}\left(\gamma_{1}\right)$ make $j$-turns about $P_{2}$ until they approach $\gamma_{1}$ in backwards time. See Fig. 2.2.

A similar procedure can be applied to label the cuts made by $W^{s}\left(\gamma_{2}\right)$ and $W^{u}\left(\gamma_{2}\right)$ with a varying Poincaré section $\mathcal{S}_{\theta_{0}}$ by $W_{\theta_{0}, j}^{s}\left(\gamma_{2}\right)$ and $W_{\theta_{0}, j}^{u}\left(\gamma_{2}\right)$, respectively, such that the label $j$ equals the number of complete turns made by the infinitesimal mass about $P_{2}$ until it approaches $\gamma_{2}$ (in positive or negative time). The threshold values of the angle $\theta$ at which the number of complete turns about $P_{2}$ changes from $(j-1)$ to $j$ are the values $2 j \pi+\theta_{2}, j \geq 1$, where $-\theta_{2}$ and $\theta_{2}$ represent the angles made by the tangent lines from $P_{2}$ to the Lyapunov orbit $\gamma_{2}$.

The successive cuts made by the invariant manifolds $W^{s, u}\left(\gamma_{i}\right)$ with the Poincaré sections are topological circles in $\mathcal{S}_{\theta_{0}}$ up to some number of turns about the primary. When the first transverse intersection of $W^{u}\left(\gamma_{i_{1}}\right)$ with $W^{s}\left(\gamma_{i_{2}}\right)$ occurs, where $i_{1}, i_{2} \in$ $\{1,2\}$, some number of turns afterwards the topological circles are destroyed (see [24]). For example, if $W_{\theta_{0}, j}^{s}\left(\gamma_{i}\right)$ intersects transversally $W_{\theta_{0}, k}^{u}\left(\gamma_{i}\right)$ at some point $P$, then the $k$-th negative iterate of $W_{\theta_{0}, j}^{s}\left(\gamma_{i}\right)$ under $\Phi_{\theta_{0}}$, which is $W_{\theta_{0}, j+k}^{s}\left(\gamma_{i}\right)$, is no longer a topological circle, but a finite union of curves open at both ends, whose ends wrap 


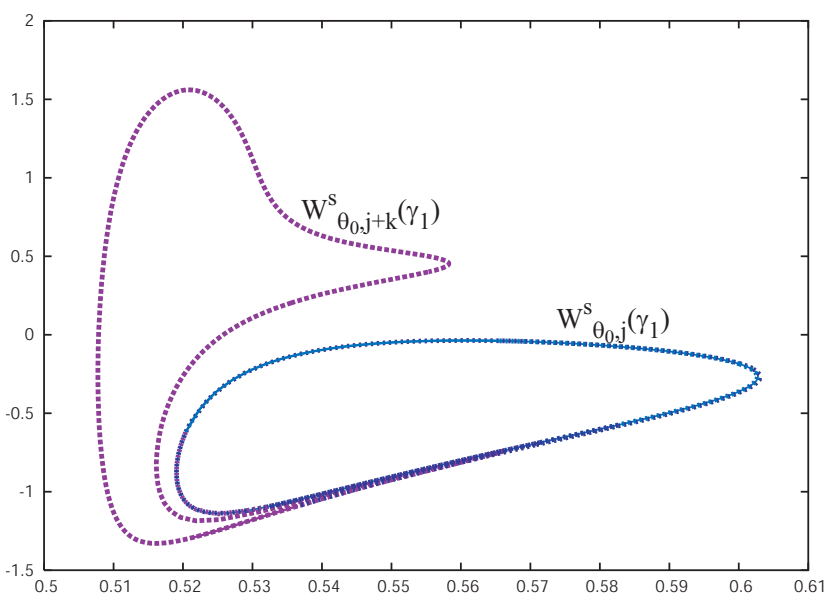

FIG. 2.3. Invariant manifold cut consisting of an open curve segments whose ends wrap some other invariant manifold cut.

around $W_{\theta_{0}, j}^{s}\left(\gamma_{i}\right)$ infinitely many times. See Fig 2.3. Also, by the Smale's Homoclinic Orbit Theorem, the iterate $\Phi_{\theta_{0}}^{-k}$ of $\Phi_{\theta_{0}}$ has a hyperbolic horseshoe near $P$; the orbits corresponding to the horseshoe display chaotic behavior that can be coded through symbolic dynamics. See [30, 24].

3. Weak Stability Boundary. In this section we give a rigorous algorithmic definition of the WSB (similar to [22]).

We consider trajectories of the infinitesimal particle with the following initial conditions:

(i) The initial position of the trajectory is on a radial segment $l(\theta)$ in the configuration space departing from $P_{2}$ and making an angle of $\theta$ with the $P_{1} P_{2}$ line, relative to the rotating system. The trajectory is assumed to start at the periapsis of an osculating ellipse around $P_{2}$, whose semi-major axis lies on $l(\theta)$ and whose eccentricity $e$ is held fixed along $l(\theta)$. The initial velocity of the trajectory is perpendicular to $l(\theta)$; there are two different such choices of initial velocities, one positive (direct motion) and one negative (retrograde motion).

(ii) The initial Keplerian energy $H_{2}$ relative to $P_{2}$ is negative, i.e., $H_{2}<0$.

(iii) Then, the motion is said to be $n$-stable if the infinitesimal mass $P_{3}$ leaves $l(\theta)$, makes $n$ complete turns about $P_{2}$ without making a complete turn around $P_{1}$, and the intersections of the trajectory with $l(\theta)$ along this trajectory are all transverse intersections and have negative Kepler energy with respect to $P_{2}$, i.e. $H_{2}<0$. The motion is otherwise said to be $n$-unstable.

In condition (i) the distance from $P_{3}$ to $P_{2}$ is given by $r=a(1-e)$, where $a$ is the semi-major axis of the osculating ellipse. The initial velocity vector fulfilling the above condition can be chosen in two ways, which only differ from one another by the sense of the vector. The initial radial velocity satisfies $\dot{r}=0$. The motion, for fixed values of the parameters $\theta$ and $e$, and for a choice of direction of the initial velocity vector depends only on the initial distance $r$. Below, we will restrict ourselves to the case of positive initial velocity. The negative initial velocity case is discussed in $[22,38]$. 
In condition (ii), the initial Kepler energy is given by (2.9) so the condition $H_{2}<0$ is automatically satisfied since $e \in[0,1)$.

Since $v=r(1+\dot{\theta})$, from (2.8) we obtain $H_{2}=\frac{1}{2} r^{2}(1+\dot{\theta})^{2}-\frac{\mu}{r}$, so the condition $H_{2}=0$ defines a 2-dimensional surface in the 3-dimensional energy manifold $\{J=C\}$. This surface separates the energy manifold into two disjoint regions where $H_{2}<0$ and $H_{2}>0$ respectively. So the condition that an intersection point of the trajectory with $l(\theta)$ has negative Kepler energy relative to $P_{2}$ means that the intersection point is in $\{J=C\} \cap\left\{H_{2}<0\right\}$.

In condition (iii), by $P_{3}$ making 1 complete turn around $P_{2}$ we mean the following. Let $\theta_{2}(t)$ be the angle made by the position vector of $P_{3}$ relative to $P_{2}$, measured continuously along the trajectory of $P_{3}$. If we consider that $P_{3}$ starts from $l(\theta)$ we have $\theta_{2}(0)=\theta$. Let $\tau_{1}$ be the smallest positive time for which $\left\|\theta_{2}\left(\tau_{1}\right)-\theta_{2}(0)\right\|=2 \pi$. Assume that the intersection of the trajectory with $l(\theta)$ at $t=\tau_{1}$ is transverse (for definition, see e.g. [9]). If such a $\tau_{1}$ exists then we say that $P_{3}$ performed 1 complete turn around $P_{2}$ in the time interval $\left[0, \tau_{1}\right]$. Similarly, let $\tau_{2}$ be the smallest positive time $\tau$ for which $\left\|\theta_{2}\left(\tau_{2}\right)-\theta_{2}\left(\tau_{1}\right)\right\|=2 \pi$. Assume that the intersection of the trajectory with $l(\theta)$ at $t=\tau_{2}$ is transverse. If such a $\tau_{2}$ exists then it means that $P_{3}$ performed 2 complete turns around $P_{2}$ in the time interval $\left[0, \tau_{2}\right]$. We can define inductively, in a similar fashion, what it means for $P_{3}$ to perform $n$ complete turns about $P_{2}$. In this definition we require that the successive intersections of the trajectory of $P_{3}$ with $l(\theta)$ are all transverse.

In the same manner, we define what means for $P_{3}$ to perform 1 complete turn about $P_{1}$. Also, if $\theta_{1}(t)$ is the angle made by the position vector of $P_{3}$ relative to $P_{1}$, measured continuously along the trajectory of $P_{3}$, if for some $\tau>0$ we have $\left\|\theta_{1}(t)-\theta_{1}(0)\right\|<2 \pi$ for all $t \in[0, \tau]$, it means that $P_{3}$ did not perform one complete turn about $P_{1}$ in the interval $[0, \tau]$.

We note that in condition (iii) the initial point of the trajectory satisfies $\dot{r}=0$ but the subsequent intersections of the trajectory with $l(\theta)$ do not have to satisfy $\dot{r}=0$. Also, between intersections, the Kepler energy $\mathrm{H}_{2}$ is allowed to be locally positive. These two conditions are imposed for applications to designing low-energy space missions and not for mathematical reasons.

In summary, condition (iii) says that a motion is said to be $n$-stable if there exist $\tau_{n}>0$ such that $P_{3}$ performed $n$ complete turns about $P_{2}$ in the interval $\left[0, \tau_{n}\right]$ and did not complete 1 turn around $P_{1}$ in the same interval.

In the above definition we require that the successive intersections of the trajectory of $P_{3}$ with $l(\theta)$ are all transverse. This makes the notion of $n$-stability under small perturbation, that is, if a trajectory is $n$-stable then any sufficiently close trajectory will also be $n$-stable. This additional restriction does not have any practical consequences in the numerical computation of the WSB, as the initial conditions whose trajectories will fail the transversality condition have zero measure.

We note that the $n$-stability condition defined as above is an open condition. This is due to the fact that $H_{2}<0$ is an open condition, and that the mapping $\theta(0) \mapsto \theta\left(\tau_{n}\right)$ on $l(\theta)$ is smooth. The stability of the number of turns about a primary, as discussed earlier, implies that if for some value of $r$ the motion is $n$-stable, then there exists a small $\delta>0$ depending on $r$ such that, for each $r^{\prime} \in(r-\delta, r+\delta)$, the corresponding motion is also $n$-stable.

We have observed numerically that for any fixed value of the eccentricity $e \in[0,1)$ all points $r \in l(\theta)$ that are sufficiently close to $P_{2}$ are $n$-stable. It seems possible that one can argue this behavior for sufficiently large positive values of the Jacobi 
energy and in some small open neighborhood of $P_{2}$ using the KAM theorem and the Nekhoroshev theorem (see $[32,37]$ ).

Thus the set of the $n$-stable points on $l(\theta)$ is an open subset of $l(\theta)$, hence it is a countable union of open intervals which we denote

$$
\mathcal{W}_{n}(\theta, e)=\bigcup_{k \geq 1}\left(r_{2 k-1}^{*}, r_{2 k}^{*}\right)
$$

with $r_{1}^{*}=0$. The points $r^{*}$ that are at the endpoints of intervals above (except for $r_{1}^{*}$ ) are $n$-unstable. In the numerical experiments, it would be impossible to detect a countable collection of stability intervals. The apparent Cantor-like structures of these sets, also noted in [22], support the possibility of having countable collection of stability intervals. In this sense, our notion of stability seems to resemble KAM stability (see e.g. [11]).

One example of unstable points are those lying on the stable manifold of the Lyapunov orbit, since they approach asymptotically the Lyapunov orbit and they never return to $l(\theta)$.

By varying the parameters $\theta$ and $e$ we obtain the following stable sets

$$
\begin{gathered}
\mathcal{W}_{n}(e)=\bigcup_{\theta \in[0,2 \pi]} \mathcal{W}_{n}(\theta, e), \\
\mathcal{W}_{n}=\bigcup_{\substack{\theta \in[0,2 \pi] \\
e \in[0,1)}} \mathcal{W}_{n}(\theta, e) .
\end{gathered}
$$

These sets are also open sets since the $n$-stability of points depends smoothly on $e$ and $\theta$. We emphasize that it is essential in the definition of the WSB that the conditions (i)-(iii) for $n$-stable points are open conditions to ensure the smooth dependence of the $n$-stability on $e$ and $\theta$.

We remark that $\mathcal{W}_{m}(e) \subset \mathcal{W}_{n}(e)$ and $\mathcal{W}_{m} \subset \mathcal{W}_{n}$ for $m>n$.

DeFINITION 3.1. The WSB of index $n$, denoted by $\mathcal{W}_{n}^{*}$, is the locus of all points $r^{*}(\theta, e)$ along the radial segment $l(\theta)$ where there is a change of stability of the initial trajectory, that is, $r^{*}(\theta, e)$ is one of the endpoints of an interval $\left(r_{2 k-1}^{*}, r_{2 k}^{*}\right)$ characterized by the fact that for all $r \in\left(r_{2 k-1}^{*}, r_{2 k}^{*}\right)$ the motion is $n$-stable, and there exist $r^{\prime}, r^{\prime \prime} \notin\left(r_{2 k-1}^{*}, r_{2 k}^{*}\right)$ arbitrarily close to $r_{2 k-1}^{*}, r_{2 k}^{*}$, respectively, for which the motion is n-unstable. Thus

$$
\mathcal{W}_{n}^{*}=\partial \mathcal{W}_{n}=\left\{r^{*}(\theta, e) \mid \theta \in[0,2 \pi], e \in[0,1)\right\} .
$$

We also denote

$$
\mathcal{W}_{n}^{*}(e)=\partial \mathcal{W}_{n}(e)=\left\{r^{*}(\theta, e) \mid \theta \in[0,2 \pi]\right\}
$$

In Fig. 3.1 we show the WSB sets $\mathcal{W}_{n}^{*}(e)$ for $n=1$ and $e=0.00, e=0.20$, $e=0.60$ and $e=0.95$, which were generated by first computing the stable sets $\mathcal{W}_{n}(e)$ and then computing the boundary sets $\mathcal{W}_{n}^{*}(e)=\partial \mathcal{W}_{n}(e)$, using a bisection method as described in [44]. We emphasize that the points in the same WSB set $\mathcal{W}_{n}^{*}(e)$ do not all have the same Jacobi constant.

REMARK 3.2. The WSB is not an invariant object for the dynamics. Moreover, the WSB is not a manifold but rather appears to be a fractal set (estimating the fractal dimension of the WSB is beyond the scope of this paper). The WSB is concerned only 

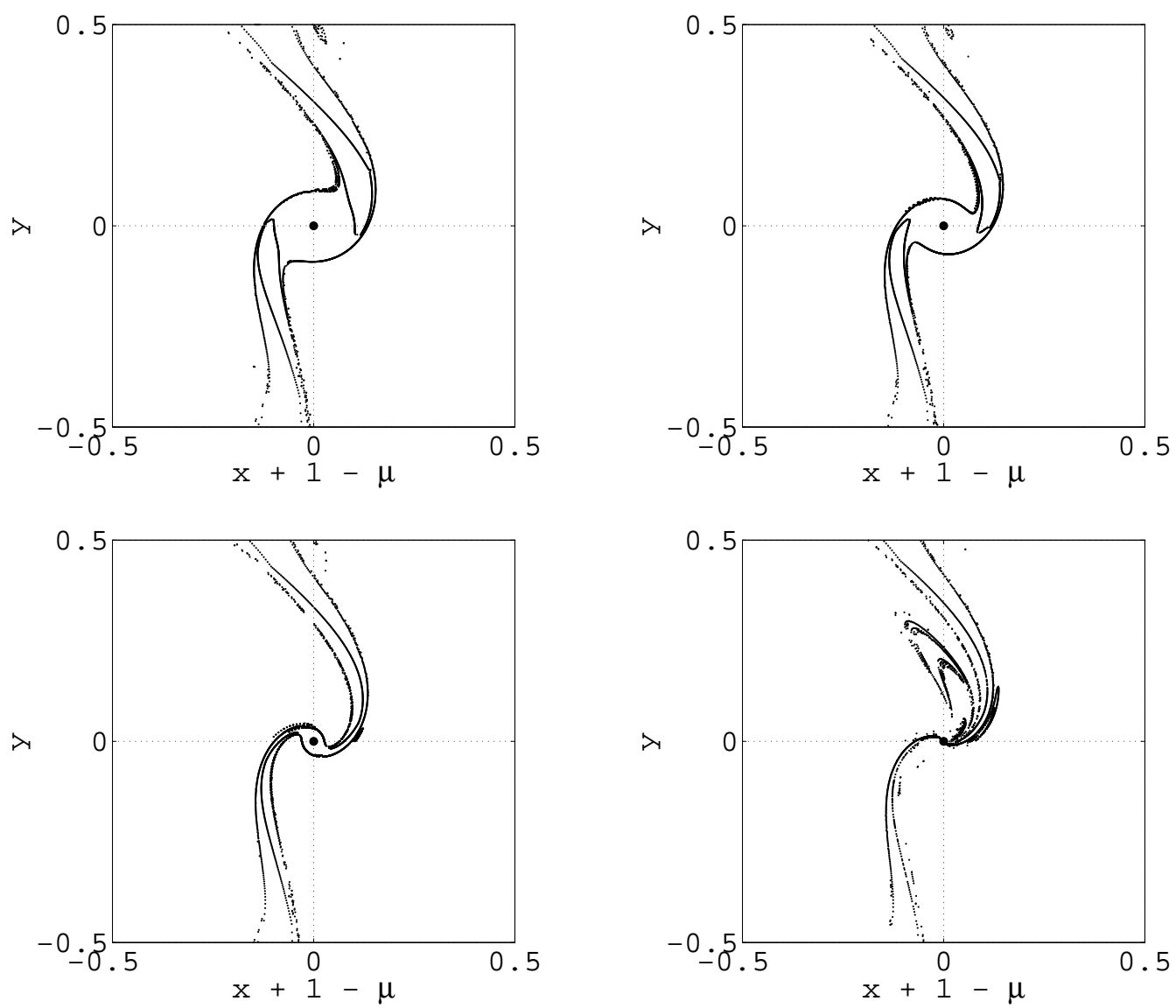

FIG. 3.1. Weak stability boundary sets $\mathcal{W}_{1}^{*}$ for eccentricities $e=0.00, e=0.20, e=0.60$, e $=0.95$.

with the behavior of a trajectory for some limited number of turns about the small primary. Counting the number of turns imposes some artificial cutoff conditions, in the sense that points with trajectories behaving rather similarly may be classified differently in terms of their stability, as they can different in the number of turns about $P_{1}$ or $P_{2}$. For example, the trajectory in Fig. 3.2 (left) is 1-stable, although it seems that it completes 1-turn around $P_{1}$. In reality, the total angle swept by the trajectory relative to $P_{1}$ is a little less than $2 \pi$. On the other hand, the trajectory in Fig. 3.2 (right) is 1-unstable although it is close to the previous orbit and it is similarly looking in the first part.

4. Weak Stability Boundary and Invariant Manifolds. In this section we describe a geometric mechanism that distinguishes $n$-stable points from $n$-unstable points and produces points in the WSB. This is based on the separatrix property of the hyperbolic invariant manifolds of the Lyapunov orbits. Then we present numerical experiments that verify the geometric mechanism.

4.1. Geometric mechanism. We give a geometric argument that, for some range of energies, the points on the stable manifold of the Lyapunov orbits about $L_{1}$ and $L_{2}$ belong to the WSB set, provided that they satisfy the zero radial velocity 

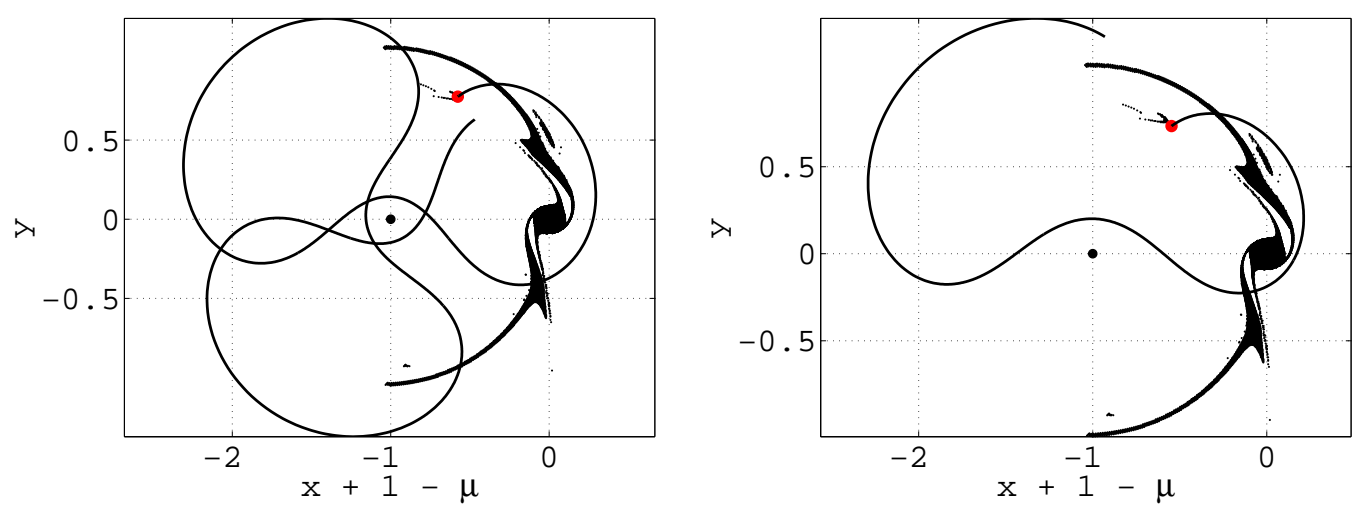

FIG. 3.2. A 1-stable trajectory that almost completes a 1-turn about $P_{1}$ (left), and a 1-unstable trajectory that returns on $l(\theta)$ with positive Kepler energy (right). The initial condition is represented by the red point.

condition and the negative Kepler energy condition relative to the small primary. This argument is based on the fact that the invariant manifolds of the Lyapunov orbits are separatrices of the energy manifold in a neighborhood of the libration points. The stable and unstable manifolds of the Lyapunov orbits are global invariant objects diffeomorphic to 2-dimensional cylinders. The trajectories inside the cylinder bounded by the stable manifold of the Lyapunov orbit about $L_{1}$ or $L_{2}$ transfer from the $P_{2^{-}}$ region to the $P_{1}$-region or to the exterior region in forward time, while the trajectories outside the cylinder bounce back to the $P_{2}$-region and remain in that region until they reach the interior of a cylinder. When these stable manifolds are cut by a plane of section that makes an angle of $\theta_{0}$ with the axis between the primaries, the points in the section that are on the stable manifolds and that satisfy the zero radial velocity condition and the negative Kepler energy condition are points in the WSB: the nearby trajectories on one side of such points are stable orbits, while the nearby trajectories on the other side of such points are unstable orbits.

The geometric argument below relates the WSB set $\mathcal{W}_{n}^{*}$ with the stable manifolds of the Lyapunov orbits about $L_{1}$ and $L_{2}$. We assume the following topological conditions on the invariant manifolds of the Lyapunov orbits. These conditions are sufficient but not necessary.

Hypothesis A. We assume that the stable manifolds and unstable manifolds of the Lyapunov orbits $\gamma_{1}$ and $\gamma_{2}$ satisfy the following topological conditions:

(i) All the trajectories on the branch of $W^{s}\left(\gamma_{1}\right)$ in the $P_{2}$-region make at least $n$-turns about $P_{2}$;

(ii) All the trajectories on the branch of $W^{u}\left(\gamma_{1}\right)$ in the $P_{1}$-region make at least 1-turn about $P_{1}$

(iii) All the trajectories on the branch of $W^{s}\left(\gamma_{2}\right)$ in the $P_{2}$-region make at least $n$-turns about $P_{2}$;

(iv) All the trajectories on the branch of $W^{u}\left(\gamma_{2}\right)$ in the exterior region make at least 1-turn about $P_{1}$.

For some values of $\mu$ and $C$ there exist analytical arguments that ensure that some of these conditions are satisfied. In the case when the mass ratio $\mu$ is very small and $(\mu, C)$ is in some open set in the $(\mu, C)$ plane, the papers [34] and [27] imply the conditions (i) and (iv) from above. We do not know of analytical results to ensure 

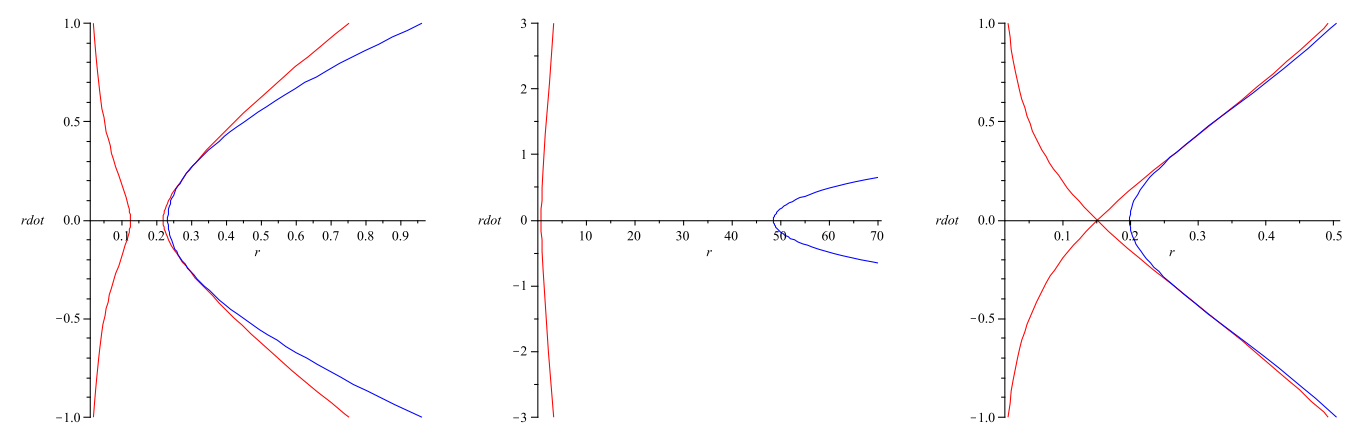

FIG. 4.1. The region $\left\{J=C_{1}\right\} \cap\left\{H_{2}<0\right\}$ in $(r, \dot{r})$-coordinates for $\theta$ fixed, where $\theta=0$, $\theta= \pm \pi / 2$, and $\theta=-\pi$. The red curves represent the boundary of $\left\{J=C_{1}\right\}$ and the blue curve represents $\left\{H_{2}=0\right\}$. In the first figure $(\theta=0)$ the closest red curve to the vertical axis corresponds to boundary of the inner Hill region, while the farthest red curve corresponds to boundary of the outer Hill region. In the second figure $(\theta= \pm \pi / 2)$ the blue curve is so distant from the vertical axis that the two red curves corresponding to the inner and outer boundaries of the Hill region cannot be distinguished in the plot. In the third figure $(\theta=\pi)$ the red curves correspond to boundaries of the two components of the inner Hill region. In each plot, the region between the vertical axis and the rightmost red curve represents $\left\{J=C_{1}\right\}$ and is always inside the region between the vertical axis and blue the curve which represents $\left\{\mathrm{H}_{2}<0\right\}$.

the conditions (ii) and (iii) for above. In the case of the Sun-Jupiter system, for some values of $C<C_{2}$, numerical and analytical methods in [30] show the existence of a symbolic dynamics which ensure the conditions (i)-(iv) from above for a certain range of values of $n$. A similar type of symbolic dynamics in the case of the Earth-Moon is more informally described in [31].

There are situations under which the conditions (i)-(iv) from above are not satisfied. For example, in the case when $W^{u}\left(\gamma_{1}\right)$ and $W^{s}\left(\gamma_{1}\right)$ collide with $P_{2}$ is possible that these manifolds intersect at some point $x<1-\mu$, so there exist trajectories on $W^{s}\left(\gamma_{1}\right)$ that do not turn around $P_{2}$, so condition (i) fails. In a similar fashion, collisions of the other branches of the invariant manifolds with either $P_{1}$ or $P_{2}$ can yield to trajectories that fail the conditions (ii), (iii) or (iv). We will exclude these situation from the geometric analysis below.

We denote by $\mathcal{W}_{n}^{*, A}$ the subset of the weak stability boundary $\mathcal{W}_{n}^{*}$ for which the conditions of Hypotheses A are satisfied. We denote by $\mathcal{W}_{n}^{*, B}$ the complementary subset of $\mathcal{W}_{n}^{*, A}$ relative to $\mathcal{W}_{n}^{*}$.

In the sequel, we will show that, for some range of energies $C>C_{\min }$,

$$
\mathcal{W}_{n}^{*, A}=\left\{(r, \dot{r}, \theta, \dot{\theta}) \in W_{\theta, n-1}^{s}\left(\gamma_{1}\right) \cup W_{\theta, n-1}^{s}\left(\gamma_{2}\right) \mid \dot{r}=0, H_{2}<0\right\},
$$

where $W_{\theta, n-1}^{s}\left(\gamma_{1}\right)$ and $W_{\theta, n-1}^{s}\left(\gamma_{2}\right)$ represent the $(n-1)$-th cut of $W^{s}\left(\gamma_{1}\right)$ and $W^{s}\left(\gamma_{2}\right)$ with the Poincaré section $\mathcal{S}_{\theta}$, where the cuts of the invariant manifolds are labeled as described in Subsection 2.4.

4.1.1. Case $C \geq C_{1}$. When $C \geq C_{1}$ the Hill's region is closed at $L_{1}$ so there are no transitions between $P_{2}$-region and the $P_{1}$-region. Inside the $P_{2}$-region the condition $H_{2}<0$ is always satisfied, see Fig. 4.1. Therefore all trajectories are $n$-stable, for any $n$.

4.1.2. Case $C_{2}<C<C_{1}$. We consider a Jacobi energy level $C_{2}<C<C_{1}$. The corresponding Hill's region is open at $L_{1}$ and closed at $L_{2}$, so transitions are possible between the $P_{2}$-region and $P_{1}$-region but not between the inner and the outer region. 
We consider the Lyapunov orbit $\gamma_{1}$ near the libration point $L_{1}$, corresponding to a fixed energy level, and the stable and unstable manifolds $W^{s}\left(\gamma_{1}\right)$ and $W^{u}\left(\gamma_{1}\right)$ of $\gamma_{1}$.

We relate the dynamics of points on the stable manifold $W^{s}\left(\gamma_{1}\right)$ to the dynamics of points in the WSB set $\mathcal{W}_{n}^{*, A}$. We label the successive cuts made by $W^{s}\left(\gamma_{1}\right)$ with $\mathcal{S}_{\theta_{0}}$ by $W_{\theta_{0}, j}^{s}\left(\gamma_{1}\right)$ in the manner as described in Subsection 2.4, such that a trajectory starting from $W_{\theta_{0}, j}^{s}\left(\gamma_{1}\right)$ completes precisely $j$-turns about $P_{2}$ before it approaches $\gamma_{1}$. By Hypothesis A (i), all the cuts with $0 \leq j \leq n$ are well defined.

It is possible that all successive cuts of the stable manifold with the section $\mathcal{S}_{\theta_{0}}$, up to the order $(n-1)$, are topological circles: $W_{\theta_{0}, 0}^{s}\left(\gamma_{1}\right), W_{\theta_{0}, 1}^{s}\left(\gamma_{1}\right), W_{\theta_{0}, 2}^{s}\left(\gamma_{1}\right)$, $\ldots, W_{\theta_{0}, n-1}^{s}\left(\gamma_{1}\right)$. If $P$ is an initial point in $\mathcal{S}_{\theta_{0}}$ inside the region bounded by $W_{\theta_{0}, 0}^{s}\left(\gamma_{1}\right)$, but sufficiently close to it, then the trajectory of $P$ will make a transfer to the $P_{1}$ region by making less than 1 complete turn about $P_{2}$. If $P$ is outside the region bounded by $W_{\theta_{0}, 0}^{s}\left(\gamma_{1}\right)$ but sufficiently close to it, the trajectory will stay in the $P_{2^{-}}$ region for at least 1-turn. Similarly, if $P$ is an initial point in $\mathcal{S}_{\theta_{0}}$ inside the region bounded by $W_{\theta_{0}, 1}^{s}\left(\gamma_{1}\right)$, then the trajectory of $P$ will make a transfer to the $P_{1}$ region by completing 1-turn about $P_{2}$ but turning less than 2 times about $P_{2}$. If $P$ is outside the region bounded by $W_{\theta_{0}, 1}^{s}\left(\gamma_{1}\right)$ but sufficiently close to it, the trajectory will stay in the $P_{2}$-region for at least 2-turns. In general, if $P$ is an initial point in $\mathcal{S}_{\theta_{0}}$ inside the region bounded by $W_{\theta_{0}, n-1}^{s}\left(\gamma_{1}\right)$ then the trajectory of $P$ will make a transfer to the $P_{1}$-region by completing $(n-1)$-turns about $P_{2}$ but turning less than $n$ times about $P_{2}$, and if $P$ is outside the region bounded by $W_{\theta_{0}, n-1}^{s}\left(\gamma_{1}\right)$, but sufficiently close to it, the trajectory will stay in the $P_{2}$-region for at least $n$-turns. A trajectory that goes to the $P_{1}$-region enters it through the interior of the region bounded by unstable manifold $W^{u}\left(\gamma_{1}\right)$. By condition (ii) of Hypothesis (A), the trajectory will make one or more complete turns around $P_{1}$ before it can return to the $P_{2}$-region. It is of course possible to also study trajectories that, once in the $P_{1}$-region, fail to make 1 complete turn around $P_{1}$ and they return to the $P_{2}$-region, but this will involve a more intricate analysis of the associated symbolic dynamics. We will avoid this analysis here.

We represent the section $\mathcal{S}_{\theta_{0}}$ in coordinates $(r, \dot{r})$. In the case when the stable manifold cut $W_{\theta_{0}, n-1}^{s}\left(\gamma_{1}\right)$ intersects transversally the axis $\dot{r}=0$ at some point $w^{*}$ of coordinates $\left(r^{*}, 0\right)$, the points $(r, 0)$ with $r$ near $r^{*}$ will be interior points to $W_{\theta_{0}, n-1}^{s}\left(\gamma_{1}\right)$ on one side of $\left(r^{*}, 0\right)$ and exterior points to $W_{\theta_{0}, n-1}^{s}\left(\gamma_{1}\right)$ on the other side of $\left(r^{*}, 0\right)$. The exterior points $(r, 0)$ are $n$-stable, provided they satisfy the Kepler energy condition $H_{2}<0$. We note that not all points in this case satisfy the negative Kepler energy condition relative to $P_{2}$. See Fig. 4.2. The interior points are $n$-unstable. The corresponding point $w^{*}$ makes the transition from $n$-stability to $n$-instability, so it is a point in the WSB. Moreover, the eccentricity of the osculating ellipse at $w^{*}$ can be computed from (2.6) as $e^{*}=\frac{\left(v^{*}\right)^{2} r^{*}}{\mu}-1$, where $v^{*}=r^{*}\left(1+\dot{\theta}_{\mid \theta=\theta_{0}}\right)$. Thus, the point $w^{*}$ is in the WSB set $\mathcal{W}_{n}^{*, A}\left(\theta_{0}, e^{*}\right)$. If the cut $W_{\theta_{0}, n-1}^{s}\left(\gamma_{1}\right)$ does not intersect the axis $\dot{r}=0$ it means that on the cut $W_{\theta_{0}, n-1}^{s}\left(\gamma_{1}\right)$ there are no WSB points from the set $\mathcal{W}_{n}^{*, A}\left(\theta_{0}, e\right)$ for any eccentricity $e$.

We remark that in the case when $\theta_{0}=0$ and $\theta_{0}=\pi$ the WSB points in the section $\mathcal{S}_{\theta_{0}}$ are symmetric homoclinic points. Indeed, the symmetry (2.3) implies that if $(r(t), \theta(t), \dot{r}(t), \dot{\theta}(t))$ is an orbit, then $(r(-t),-\theta(-t),-\dot{r}(-t), \dot{\theta}(-t))$ is also an orbit. Thus, an orbit that intersects $\mathcal{S}_{\theta_{0}}$ with $\dot{r}=0$, for $\theta_{0}=0$ or $\theta_{0}=\pi$, is a symmetric orbit. Since the stable and unstable manifolds are also symmetric, a point in $W_{\theta_{0}, n-1}^{s}\left(\gamma_{1}\right)$ with $\dot{r}=0$ is a symmetric homoclinic point provided $\theta_{0}=0$ or $\theta_{0}=\pi$.

It is possible that not all the cuts made by the stable manifold with the plane of 

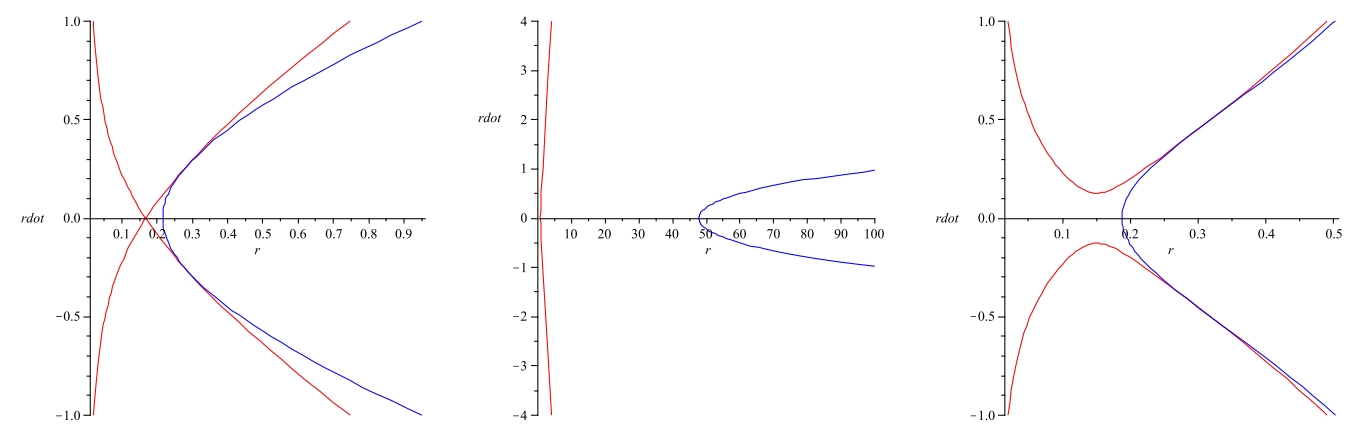

FIG. 4.2. The region $\left\{J=C_{2}\right\} \cap\left\{H_{2}<0\right\}$ in $(r, \dot{r})$-coordinates for $\theta$ fixed, where $\theta=0$, $\theta= \pm \pi / 2$, and $\theta=-\pi$. The red curve represents the boundary of $\left\{J=C_{2}\right\}$ and the blue curve represents $\left\{H_{2}=0\right\}$. The region between the vertical axis and the rightmost red curve represents $\left\{J=C_{2}\right\}$ and is always inside the region between the vertical axis and blue curve which is $\left\{H_{2}<0\right\}$.

section are topological circles, as noted in Subsection 2.4. If $W_{\theta_{0}, j}^{s}\left(\gamma_{1}\right)$ is a topological circle in $\mathcal{S}_{\theta_{0}}$ that intersects $W_{\theta_{0}, k}^{u}\left(\gamma_{1}\right)$ which is also a topological circle, then any intersection point is a homoclinic point which makes $j+k-1$ turns around $P_{2}$, and the region bounded by both $W_{\theta_{0}, j}^{s}\left(\gamma_{1}\right)$ and $W_{\theta_{0}, k}^{u}\left(\gamma_{1}\right)$ consists of trajectories that make transitions to the $P_{1}$-region in negative time, after $j+k-1$ turns about $P_{2}$. Thus $W_{\theta_{0}, j+k}^{s}\left(\gamma_{1}\right)$ is no longer a topological circle; it consists of a finite number of curves open at both ends that wrap asymptotically around $W_{\theta_{0}, j}^{s}\left(\gamma_{1}\right)$ infinitely many times. Although the topological circle property is lost, we can still distinguish between 'interior' points to the region bounded by $W_{\theta_{0}, j+k}^{s}\left(\gamma_{1}\right)$ and 'exterior' points (from the point of view of separatrix property): the interior points are precisely the image under $\Phi_{\theta_{0}}^{-k}$ of the points inside the region bounded by $W_{\theta_{0}, j}^{s}\left(\gamma_{1}\right)$ and outside the region bounded by $W_{\theta_{0}, k}^{u}\left(\gamma_{1}\right)$ in $\mathcal{S}_{\theta_{0}}$. The conclusion is that the points interior to the region bounded by $W_{\theta_{0}, j+k}^{s}\left(\gamma_{1}\right)$ correspond to trajectories that leave the $P_{2}$-region in $(j+k-1)$-turns, while the points exterior to the region bounded by $W_{\theta_{0}, j+k}^{s}\left(\gamma_{1}\right)$ correspond to trajectories that remain in the $P_{2}$-region for at least $(j+k)$-turns.

The same type of argument can be carried out when a broken topological circle, like $W_{\theta_{0}, j+k}^{s}\left(\gamma_{1}\right)$ from above, intersects some unstable manifold cut $W_{\theta_{0}, \ell}^{u}\left(\gamma_{1}\right)$. All the points interior to the region bounded by both $W_{\theta_{0}, j+k}^{s}\left(\gamma_{1}\right)$ and $W_{\theta_{0}, \ell}^{u}\left(\gamma_{1}\right)$ correspond to trajectories that make a transition to the $P_{1}$-region in negative time, after $(j+k+\ell-1)$ turns about $P_{2}$. The return $W_{\theta_{0}, j+k+\ell}^{s}\left(\gamma_{1}\right)$ of $W_{\theta_{0}, j+k}^{s}\left(\gamma_{1}\right)$ to $\mathcal{S}_{\theta_{0}}$ under $\Phi_{\theta_{0}}^{-\ell}$ consists of a finite number of curves open at both ends that wrap asymptotically around the components of $W_{\theta_{0}, j+k}^{s}\left(\gamma_{1}\right)$ infinitely many times. The interior points to the region bounded by $W_{\theta_{0}, j+k+\ell}^{s}\left(\gamma_{1}\right)$ are the image under $\Phi_{\theta_{0}}^{-\ell}$ of the points interior to the region bounded by $W_{\theta_{0}, j+k}^{s}\left(\gamma_{1}\right)$ and exterior to $W_{\theta_{0}, \ell}^{u}\left(\gamma_{1}\right)$ in $\mathcal{S}_{\theta_{0}}$. The interior points to the region bounded by $W_{\theta_{0}, j+k+\ell}^{s}\left(\gamma_{1}\right)$ correspond to trajectories that leave the $P_{2}$-region in $(j+k+\ell-1)$-turns, while the points exterior to the region bounded by $W_{\theta_{0}, j+k+\ell}^{s}\left(\gamma_{1}\right)$ correspond to trajectories that remain in the $P_{2}$-region for at least $(j+k+\ell)$-turns.

The conclusion is that the transverse intersection points between the stable manifold cut $W_{\theta_{0}, n-1}^{s}\left(\gamma_{1}\right)$ with the axis $\dot{r}=0$ in $\mathcal{S}_{\theta_{0}}$ are points in the WSB set $\mathcal{W}_{n}^{*}\left(\theta_{0}, e^{*}\right)$ for some $e^{*}$, regardless on whether $W_{\theta_{0}, n-1}^{s}\left(\gamma_{1}\right)$ is a topological circle or not.

4.1.3. Case $C_{\min }<C<C_{2}$. We consider a Jacobi energy level $C_{\min }<C<C_{2}$ for some $C_{\min }$ large enough so that the Lyapunov orbits around $L_{1}$ and $L_{2}$ do not collide with $P_{2}$. A safe choice is assuming $C_{\min }=3.15$. The corresponding Hill's 

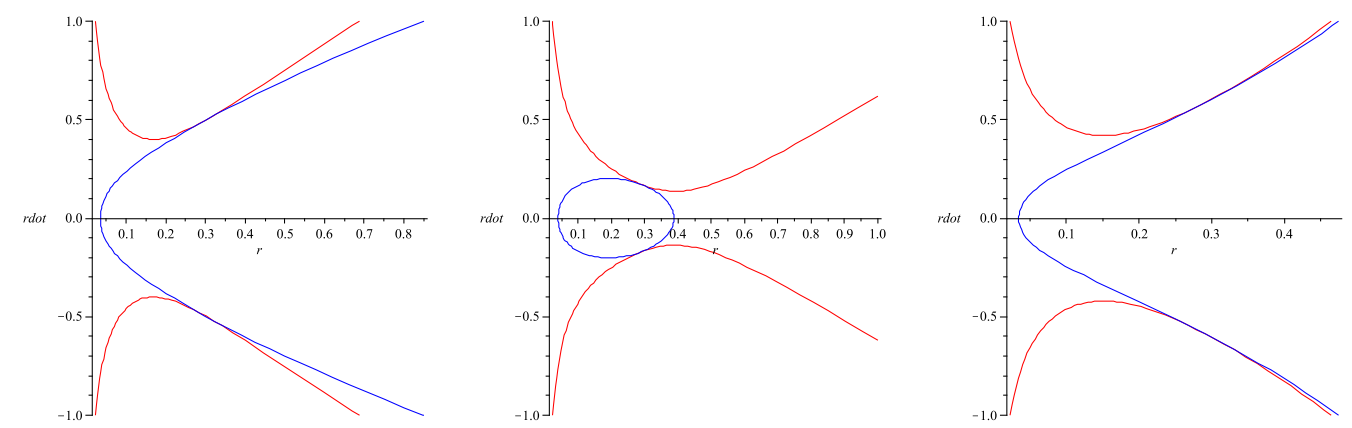

FIG. 4.3. The region $\left\{J=C_{3}\right\} \cap\left\{H_{2}<0\right\}$ in $(r, \dot{r})$-coordinates for $\theta$ fixed, where $\theta=0$, $\theta= \pm \pi / 2$, and $\theta=-\pi$. The red curve represents the boundary of $\left\{J=C_{3}\right\}$ and the blue curve represents $\left\{H_{2}=0\right\}$. The region between the vertical axis and the rightmost red curve represents $\left\{J=C_{3}\right\}$ and is always inside the region between the vertical axis and blue curve which is $\left\{H_{2}<0\right\}$.

region is open at both $L_{1}$ and $L_{2}$, so transitions are possible between $P_{2}$-region and $P_{1}$-region and also between the inner region and the outer region. In the numerical computations in Subsection 2.3 the value of $C_{\min }$ will be restricted by our numerical methods to compute the Lyapunov orbits and their invariant manifolds.

We consider the Lyapunov orbits $\gamma_{1}$ near the libration point $L_{1}$ and $\gamma_{2}$ near the libration point $L_{2}$, corresponding to a fixed energy level. We also consider the stable and unstable manifolds $W^{s}\left(\gamma_{1}\right)$ and $W^{u}\left(\gamma_{1}\right)$ of $\gamma_{1}$, and the stable and unstable manifolds $W^{s}\left(\gamma_{2}\right)$ and $W^{u}\left(\gamma_{2}\right)$ of $\gamma_{2}$. The stable manifolds $W^{s}\left(\gamma_{1}\right)$ and $W^{s}\left(\gamma_{2}\right)$ never intersect, and the unstable manifolds $W^{u}\left(\gamma_{1}\right)$ and $W^{u}\left(\gamma_{2}\right)$ never intersect as well. On the other hand, the stable manifolds can pass very close to one another, and so can the unstable manifolds.

We label the successive cuts made by $W^{s}\left(\gamma_{1}\right)$ with the Poincare section $\mathcal{S}_{\theta_{0}}$ by $W_{\theta_{0}, j}^{s}\left(\gamma_{1}\right)$ in the manner as described in Subsection 2.4, such that a trajectory starting from $W_{\theta_{0}, j}^{s}\left(\gamma_{1}\right)$ completes precisely $j$-turns about $P_{2}$ before it approaches $\gamma_{1}$, and the successive cuts made by $W^{s}\left(\gamma_{2}\right)$ with $\mathcal{S}_{\theta_{0}}$ by $W_{\theta_{0}, j}^{s}\left(\gamma_{2}\right)$, such that a trajectory starting from $W_{\theta_{0}, j}^{s}\left(\gamma_{2}\right)$ completes precisely $j$-turns about $P_{2}$ before it approaches $\gamma_{2}$. By Hypothesis A (i) and (iii), all the cuts with $0 \leq j \leq n$ are well defined.

These successive cuts made by the stable manifolds with the surface of section are topological circles or broken circles, but in either case they bound regions with well defined interior points (in the sense of the separatrix property), as explained in the Subsection 4.1.3. If an initial point $P$ in $\mathcal{S}_{\theta_{0}}$ is an interior point to the region bounded by $W_{\theta_{0}, n-1}^{s}\left(\gamma_{1}\right)$ then the trajectory of $P$ will complete $(n-1)$-turns about $P_{2}$ and make a transfer to the $P_{1}$ region. Once in the $P_{1}$ region, it will complete at least 1-turn about $P_{1}$ before it can return to the $P_{2}$ region, due to Hypothesis A (ii). On the other hand, if $P$ is outside the region bounded by $W_{\theta_{0}, n-1}^{s}\left(\gamma_{1}\right)$ but sufficiently close to it then the trajectory will stay in the $P_{2}$-region for at least $n$-turns.

Similarly, if an initial point $P$ in $\mathcal{S}_{\theta_{0}}$ is an interior point to the region bounded by $W_{\theta_{0}, n-1}^{s}\left(\gamma_{2}\right)$ then the trajectory of $P$ will complete $(n-1)$-turns about $P_{2}$ and make a transfer to the exterior region. Once the trajectory is in the exterior region, it will complete at least 1-turn about $P_{1}$ before it can return to the interior region, according to Hypothesis A (iv). If $P$ is outside the region bounded by $W_{\theta_{0}, n-1}^{s}\left(\gamma_{2}\right)$ but sufficiently close to it the trajectory will stay in the $P_{2}$-region for at least $n$-turns.

To detect the WSB points we consider the transverse intersection points $w^{*}$ of 
the stable manifold cuts $W_{\theta_{0}, n-1}^{s}\left(\gamma_{1}\right) \cup W_{\theta_{0}, n-1}^{s}\left(\gamma_{2}\right)$ with the axis $\dot{r}=0$ in $\mathcal{S}_{\theta_{0}}$. These intersection points are points in the WSB set $\mathcal{W}_{n}^{*}\left(\theta_{0}, e\right)$ for some $e^{*}$ provided they satisfy the following conditions: the points near $w^{*}$ interior to $W_{\theta_{0}, n-1}^{s}\left(\gamma_{1}\right) \cup W_{\theta_{0}, n-1}^{s}\left(\gamma_{2}\right)$ satisfy the Kepler energy condition $H_{2}<0$. We note that not all points in this case satisfy the negative Kepler energy condition relative to $P_{2}$ (see Fig. 4.3). The points near $w^{*}$ exterior to $W_{\theta_{0}, n-1}^{s}\left(\gamma_{1}\right) \cup W_{\theta_{0}, n-1}^{s}\left(\gamma_{2}\right)$ that leave the $P_{2}$-region make at least 1 full turn about $P_{1}$ inside the $P_{1}$-region or in the exterior region before they may eventually return to the $P_{2}$-region. In addition, the points $w^{*}$ as above that are found in a plane of section $\mathcal{S}_{\theta_{0}}$ with $\theta_{0}=0$ or $\theta_{0}=\pi$ are symmetric homoclinic points.

REMARK 4.1. The key geometrical argument in the above is that the stable and unstable manifolds of a Lyapunov orbit are 2-dimensional invariant manifolds that separate the 3-dimensional energy manifold. It shows that, for a fixed energy level, the corresponding WSB points lie on the stable manifold of the Lyapunov orbit. However, the WSB set consists of points on different energy manifolds. The WSB set itself is not an invariant manifold and does not have the separatrix property. As the WSB is not restricted to an energy manifold, the WSB concept can be extended to other situations where energy manifolds are not invariant or where stable invariant manifolds of periodic orbits cannot be defined.

REMARK 4.2. In the case when $W_{\theta_{0}, n-1}^{s}\left(\gamma_{i}\right)$ is a broken topological circle consisting of infinitely many open curves that wrap around asymptotically about some $W_{\theta_{0}, m}^{s}\left(\gamma_{1}\right)$ with $m<n-1$, each intersection point $w_{j}^{*}$ between such a component with the axis $\dot{r}=0$ in $\mathcal{S}_{\theta_{0}}$ yields a point in the WSB set $\mathcal{W}_{n}^{*}\left(\theta_{0}, e_{j}^{*}\right)$ for some $e_{j}^{*}$. We emphasize here that the eccentricities of the osculating orbits corresponding to the points $w_{j}^{*}$ are all different, although very close to one another, so these points belong to different WSB sets $\mathcal{W}_{n}^{*, A}(e)$ of different eccentricities $e$, as those in Fig. 3.1. However, a small change in the energy level of $\gamma_{i}$ yields a small change in the positions $w_{j}^{*}$ and hence of the corresponding eccentricities $e_{j}^{*}$ of the osculating ellipses. Thus, through a sequence of successive small perturbations of the energy level we can slightly move each point $w_{j}^{*}$, one at a time, so that all resulting points will have the same eccentricity for their osculating ellipses (with the resulting points $w_{j}^{*}$ at different energy levels). Here we recall that the WSB sets $\mathcal{W}_{n}^{*, A}(e)$ contains points not necessarily at the same energy level. This perturbation argument supports the possibility of having a countably infinite collection of stable intervals $\left(r_{2 k-1}^{*}, r_{2 k}^{*}\right)$ in (3.1), and could perhaps explain some of the fine Cantor set-like structures that are visible in some regions of the WSB sets. See the plots in Fig. 3.1.

REMARK 4.3. It seems possible to analyze the WSB in the context of the planar Hill problem. The structure of invariant manifolds of periodic orbits near the equilibrium points is similar to that in the PCRTBP $([42])$. It is expected that the same relationship between the WSB and the stable invariant manifold of periodic orbits holds in the planar Hill problem as in the PCRTBP.

4.2. Numerical experiments. We describe a numerical algorithm to test that the points on the WSB coincide with the points on the stable manifolds of the Lyapunov orbits which satisfy the zero radial velocity condition and the negative Kepler energy condition relative to the small primary. This algorithm is centered on the idea that the hyperbolic invariant manifolds are separatrices of the energy manifold. We illustrate this algorithm with several examples and we discuss the results.

In Subsection 4.1 we provided a geometric argument to show that, for some range 
of energies,

$$
\left\{(r, \dot{r}, \theta, \dot{\theta}) \in W_{\theta, n-1}^{s}\left(\gamma_{1}\right) \cup W_{\theta, n-1}^{s}\left(\gamma_{2}\right) \mid \dot{r}=0, H_{2}<0\right\}
$$

is the subset $\mathcal{W}_{n}^{*, A}$ of $\mathcal{W}_{n}^{*}$, consisting of the WSB points for which the invariant manifolds of the Lyapunov orbits by $L_{1}$ and $L_{2}$ satisfy some topological conditions described by Hypothesis A. However, Hypothesis A is a sufficient condition for a point in $\mathcal{W}_{n}^{*}$ to be in the set (4.2) but not necessary. It is possible that the stable and unstable manifolds of $\gamma_{1}$ and $\gamma_{2}$ as a whole fail Hypothesis A, but nevertheless individual trajectories on those manifolds exhibit behavior consistent with the conditions of $\mathrm{Hy}-$ pothesis $\mathrm{A}$. The numerical experiments below detect the points in $\mathcal{W}_{n}^{*}$ that are also in the set (4.2); we will call these points of type A. There are also points in in $\mathcal{W}_{n}^{*}$ that are not in the set (4.2); we will call these points of type B. The set of all points of type A includes $\mathcal{W}_{n}^{*, A}$.

In the sequel, we will compute numerically $\mathcal{W}_{n}^{*}$ and we will identify the subset of points of type A, as the WSB points that satisfy (4.2) modulo some margin of error. The numerical algorithm consists of the following steps.

(1) We compute numerically the WSB set $\mathcal{W}_{n}^{*}(e)$ for a fixed number of turns $n$ and for a fixed eccentricity $e$. We limit to points with energy $C_{\min }<C<C_{2}$. This computation is done in two steps. In the first step we compute the $n$-stable set $\mathcal{W}_{n}(e)$. For this, we divide the eccentricity range into a grid of values $e=\{0,0.05, \ldots, 0.95\}$. We fix one value of $e$ at a time and we compute the corresponding $n$-stable orbits as follows. We choose a range of radius values around $P_{2}$ and we approximate this range by a grid of values $r \in\left\{0,2 \cdot 10^{-3}, \ldots, 1.5\right\}$. We also approximate the angular range $\theta \in[0,2 \pi]$ around $P_{2}$ by a grid of values $\theta \in\left\{0,2 \pi \cdot 10^{-3}, \ldots, 2 \pi\right\}$. For each fixed $\theta$ from the grid, we consider an initial point $(r, \theta)$ with $r$ from the grid, and an initial velocity perpendicular to the position vector $(r, \theta)$, of size $v$ given by (2.6). We integrate this initial condition forward in time in polar coordinates with respect to both $P_{2}$ and $P_{1}$. The evolution in time of the angle swept around $P_{2}$ and $P_{1}$ is tracked and the negative Kepler energy relative to $P_{2}$ is verified at the intersection points with $l(\theta)$. The point is redeemed as $n$ stable or $n$-unstable according to the algorithmic definition in Section 3 . The coordinates $(r, \dot{r}, \theta, \dot{\theta})$, and the Jacobi constant $C$ of each $n$-stable point are recorded. The Jacobi constant is also given by (2.7). In the second step, the WSB is computed as the boundary set $\mathcal{W}_{n}^{*}(e)=\partial\left(W_{n}(e)\right)$ of the $n$-stable set, using a bisection algorithm. The output is a data set of points $w_{i}^{*}$ with their coordinates and Jacobi constants. The details of this computation are given in [44].

(2) For each fixed value of the eccentricity $e$, the points $w_{i}^{*}$ of the WSB set $\mathcal{W}_{n}^{*}(e)$ are analyzed one at a time with respect to their relationship with the stable manifolds of the Lyapunov orbits. Each point is retrieved from the data set together with its coordinates $\left(r_{i}^{*}, \dot{r}_{i}^{*}, \theta_{i}^{*}, \dot{\theta}_{i}^{*}\right)$ and the corresponding Jacobi constant $C_{i}$.

(3) For each Jacobi constant $C_{i}$ the Lyapunov orbits $\gamma_{1}$ and $\gamma_{2}$ are computed numerically.

(4) The stable manifolds $W^{s}\left(\gamma_{1}\right)$ and $W^{s}\left(\gamma_{2}\right)$ are integrated numerically in polar coordinates relative to $P_{2}$.

(5) The stable manifolds $W^{s}\left(\gamma_{1}\right)$ and $W^{s}\left(\gamma_{2}\right)$ are cut with the Poincaré section $\mathcal{S}_{\theta_{i}^{*}}$, where $\theta_{i}^{*}$ is the angle coordinate associated with the point $r_{i}^{*}$. The 
Poincaré section $\mathcal{S}_{\theta_{i}^{*}}$ is represented in the coordinates $(r, \dot{r})$. The points in the $(n-1)$-th cut of $W^{s}\left(\gamma_{1}\right)$ and $W^{s}\left(\gamma_{2}\right)$ with the Poincaré section are retained.

(6) The intersection points between $W^{s}\left(\gamma_{1}\right) \cup W^{s}\left(\gamma_{2}\right)$ with the axis $\dot{r}=0$ in $\mathcal{S}_{\theta_{i}^{*}}$ are computed numerically. As in general there might be several such intersection points, only one of them, $w_{i}^{* *}=\left(r_{i}^{* *}, 0, \theta_{i}^{* *}, \dot{\theta}_{i}^{* *}\right)$ will have the eccentricity of the corresponding osculating ellipse equal to $e$, modulo some small margin of error. Moreover, we select only those points $w_{i}^{* *}$ that have negative Kepler energy relative to $P_{2}$

(7) We select the points $w_{i}^{*}$ that are within a small margin of error from the corresponding points $w_{i}^{* *}$. These are the points of type A and they include the set $\mathcal{W}_{n}^{*, A}$, the set of points in $\mathcal{W}_{n}^{*}$ that satisfy the Hypothesis A. The complementary set are the points of type B. The practical method to select the points of type $\mathrm{A}$ is the following. We compute the Euclidean distance in phase space between the original point $w_{i}^{*}$, computed through the bisection algorithm, and the new point $w_{i}^{* *}$, computed as the cuts between the stable manifold and the axis $\dot{r}=0$ in the the Poincaré section:

$$
d=\left\|w_{i}^{*}-w_{i}^{* *}\right\| \text {. }
$$

We only select those points $w_{i}^{* *}$ for which the distance from the corresponding $w_{i}^{*}$ is within a tolerance of $\delta=10^{-3}$. (Since our numerical procedures are automatic procedures that explore a large number of points in the WSB sets, generate the Lyapunov orbits for the energy level of each point on the WSB, integrate the stable manifolds of these Lyapunov orbits, compute and count the cuts of these manifolds with Poincaré sections of prescribed angles, and detect the intersection of these cuts with $\dot{r}=0$, we found that this indirect procedure to identify the subset $\mathcal{W}_{n}^{*, A}$ is computationally effective. It is nevertheless true that these procedures have intrinsic limitations by the choice of algorithm for each component numerical routine, and by numerical errors.)

(8) To the selected points $w_{i}^{* *}$ found in the intersections between the stable manifold and the axis $\dot{r}=0$ in the Poincare section, we apply a test to verify numerically that they make the transition between $n$-stability and $n$ instability. This test confirms that the selected points $w_{i}^{* *}$ are in the WSB set $\mathcal{W}_{n}^{*}(e)$. For a given point $w_{i}^{* *}=\left(r_{i}^{* *}, 0, \theta_{i}^{* *}, \dot{\theta}_{i}^{* *}\right)$, two nearby points $w_{i}^{\prime}$ and $w_{i}^{\prime \prime}$ are chosen on the radial line $\theta=\theta_{i}$, of coordinates $w_{i}^{\prime}=\left(r_{i}^{\prime}, \dot{r}_{i}^{\prime}, \theta_{i}^{\prime}, \dot{\theta}_{i}^{\prime}\right)$ and $w_{i}^{\prime \prime}=\left(r_{i}^{\prime \prime}, \dot{r}_{i}^{\prime \prime}, \theta_{i}^{\prime \prime}, \dot{\theta}_{i}^{\prime \prime}\right)$. The coordinates are chosen so that $w_{i}^{\prime}$ and $w_{i}^{\prime \prime}$ are in the same Poincaré section $\mathcal{S}_{\theta_{i}^{*}}$ as $w_{i}^{* *}$ and their associated osculating ellipse have the same eccentricity $e$ as $w_{i}^{* *}$, i.e. $r_{i}^{\prime}=r_{i}^{* *}+\epsilon, \dot{r}_{i}^{\prime}=0, \theta_{i}^{\prime}=\theta_{i}^{*}$, $\dot{\theta}_{i}^{\prime}=\sqrt{\frac{\mu(1+e)}{\left(r_{i}^{\prime}\right)^{3}}}-1$, and $r_{i}^{\prime \prime}=r_{i}^{* *}-\epsilon, \dot{r}_{i}^{\prime \prime}=0, \theta_{i}^{\prime \prime}=\theta_{i}^{*}, \dot{\theta}_{i}^{\prime \prime}=\sqrt{\frac{\mu(1+e)}{\left(r_{i}^{\prime \prime}\right)^{3}}}-1$, where $\epsilon=10^{-8}$. The points $w_{i}^{\prime}$ and $w_{i}^{\prime \prime}$ are integrated forward in time in polar coordinates with respect to both $P_{2}$ and $P_{1}$. The evolution in time of the angle swept around $P_{2}$ and $P_{1}$ is tracked, and the negative Kepler energy condition relative to $P_{2}$ is verified at the intersection points with $l\left(\theta_{i}^{*}\right)$. This test is assessed as successful if one of the points $w_{i}^{\prime}, w_{i}^{\prime \prime}$ is redeemed as $n$-stable and the other one is redeemed as $n$-unstable. In the numerical examples explored, all selected points are found to satisfy this condition.

EXAMPLE 4.4. An example of applying the algorithm is illustrated in Fig. 4.4. We select a point $w_{i}^{*}$ from the WSB set $\mathcal{W}_{1}^{*}(0)$ in a Poincaré section $\mathcal{S}_{\theta_{i}^{*}}, \theta_{i}^{*} \simeq$ $3 \pi / 4$. The Lyapunov orbits $\gamma_{1}$ and $\gamma_{2}$ for the Jacobi constant $C_{i}=3.1645669491$ 

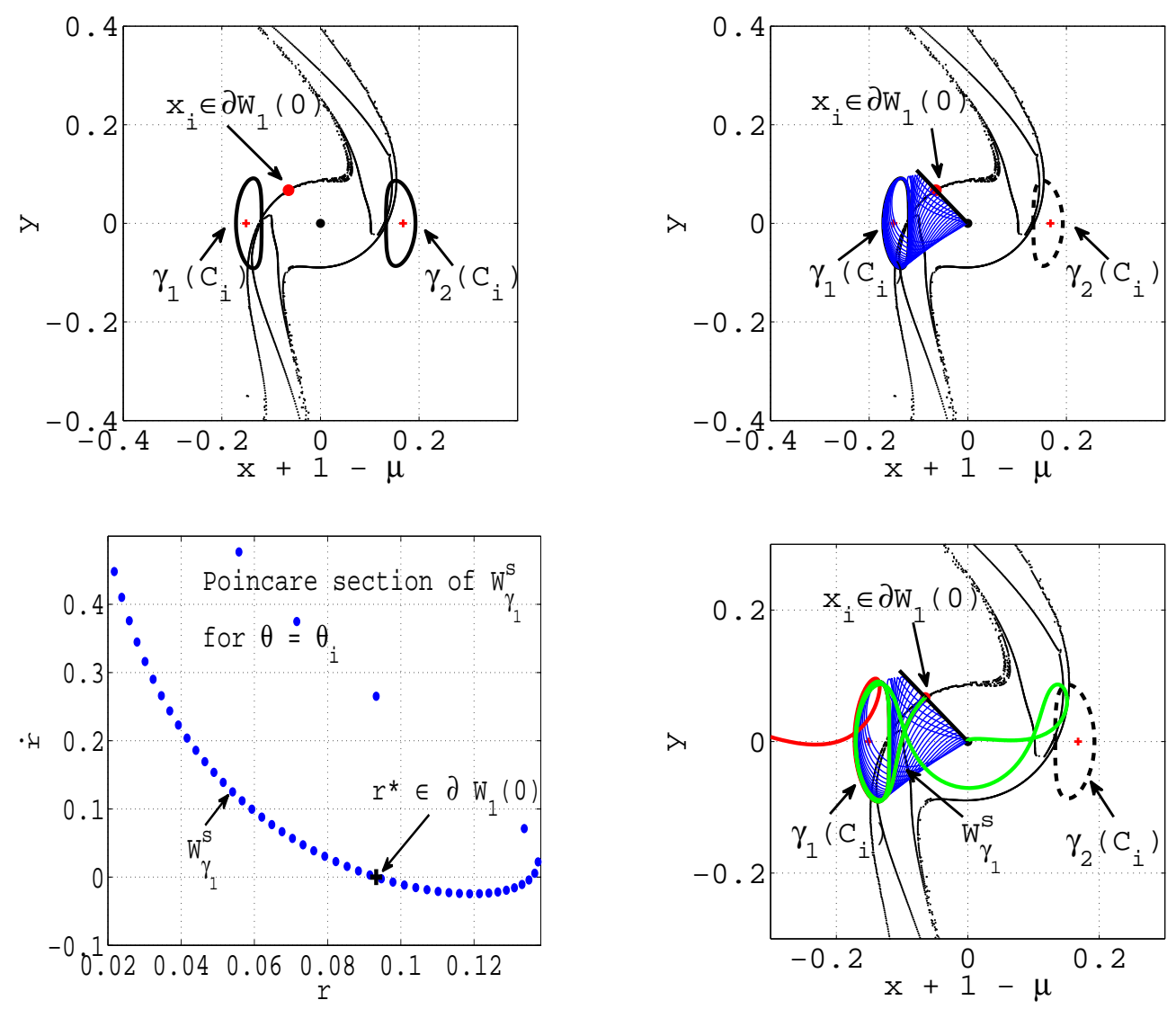

FIG. 4.4. From left to right: (a) a point in the WSB and the Lyapunov orbits for the corresponding energy level, (b) the stable manifold of the Lyapunov orbit about $L_{1}$, (c) the cut made by the stable manifold with the Poincaré section and its intersection with $\dot{r}=0,(d)$ forward integration of two initial points near the intersection of the stable manifold with $\dot{r}=0$.

corresponding to the point $w_{i}$ are computed numerically. The stable manifold $W^{s}\left(\gamma_{1}\right)$ is cut with the Poincaré section $\mathcal{S}_{\theta_{i}^{*}}$. This cut is $W_{\theta_{i}^{*}, 0}^{s}\left(\gamma_{1}\right)$, as the points in the cut complete 0 -turns about $P_{2}$ before approaching $\gamma_{1}$. The intersection between $W^{s}\left(\gamma_{1}\right)$ and the axis $\dot{r}=0$ in $\mathcal{S}_{\theta_{i}^{*}}$ is identified. In the same plot, the original point $w_{i}^{*}$ is shown. The intersection $w_{i}^{* *}$ between $W_{\theta_{i}^{*}, 0}^{s}\left(\gamma_{1}\right)$ and the axis $\dot{r}=0$ in $\mathcal{S}_{\theta_{i}^{*}}$ and the original point $w_{i}^{*}$ almost overlap in the plot. The points $w_{i}^{*}$ and $w_{i}^{* *}$ are within a $\delta$-tolerance, so the point $w_{i}^{*} \approx w_{i}^{* *}$ is of type A. To apply the test described in (8), the intersection point between $W^{s}\left(\gamma_{1}\right)$ and the axis $\dot{r}=0$ is fixed and two nearby points in $\mathcal{S}_{\theta_{i}^{*}}$, with the eccentricities of the associated osculating ellipses chosen to be $e=0$, are chosen. The two nearby points are integrated forward in time. The trajectories of the two points show that one is 1-stable and the other one is 1-unstable, which confirms our test.

EXAMPLE 4.5. Another example of applying this algorithm is illustrated in Fig. 4.5. This is similar to the previous example with the only difference being that the WSB point selected matches a point on the stable manifold $W^{s}\left(\gamma_{2}\right)$ of the Lyapunov orbit about $L_{2}$ for the Jacobi constant $C_{i}=3.1539757951$. 

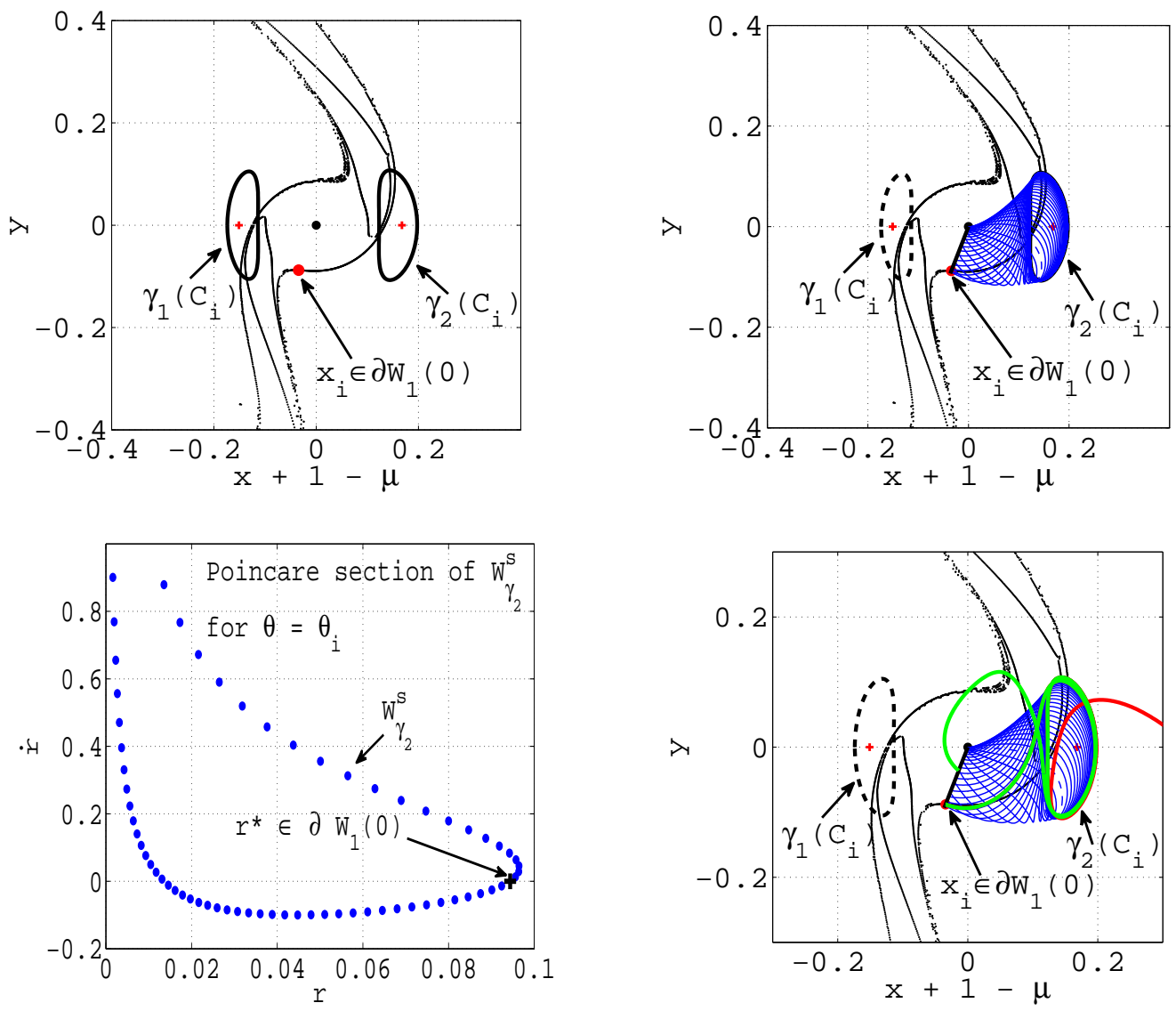

FiG. 4.5. From left to right: (a) a point in the WSB and the Lyapunov orbits for the corresponding energy level, (b) the stable manifold of the Lyapunov orbit about $L_{2}$, (c) the cut made by the stable manifold with the Poincaré section and its intersection with $\dot{r}=0,(d)$ forward integration of two initial points near the intersection of the stable manifold with $\dot{r}=0$.

EXAMPLE 4.6. We restrict to an energy range $C \in\left[C_{\min }, C_{2}\right]$, and we consider the WSB sets $\mathcal{W}_{n}^{*}(e)$ with $n=1,2$ and $e=0.4$. We match the points $w_{i}^{*}$ obtained from the algorithmic definition of the WSB as in (1) with the points $w_{i}^{* *}$ obtained from the $(n-1)$-th cut of $W^{s}\left(\gamma_{1}\right)$ or $W^{s}\left(\gamma_{2}\right)$ with Poincaré section $\mathcal{S}^{\theta_{i}^{*}}$ intersected with the axis $\dot{r}=0$ inside this section, as described in (6).

In Fig. 4.6 the displayed curve represents the points $(r, \theta)$ for which $J=C_{\min }$ for $e$ fixed (see (2.7)). The gray points are the points $w_{i}^{*}$ found by the algorithmic definition as in (1). The points $w_{i}^{*}$ in the interior region bounded by the curve are those that satisfy the energy restriction $C \in\left[C_{\min }, C_{2}\right]$. The points displayed in black are the points $w_{i}^{* *}$ that are obtained as in (7). These are the points of type A and they contain $\mathcal{W}_{n}^{*, A}$ Thus, for these points we have a matching between the WSB points obtained through the algorithmic definition and the points obtained through the invariant manifold approach.

There are a few WSB points in the interior region bounded by the energy condition curve which do not match with points on the stable manifolds. These are the points of type B. These are points whose trajectories experience close encounters or 

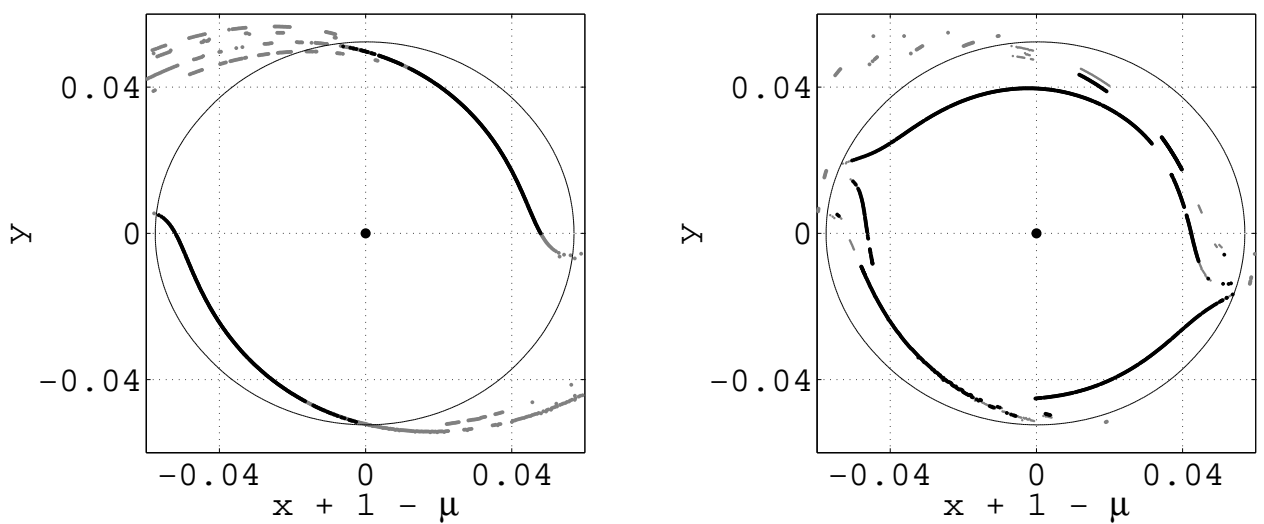

FIG. 4.6. Set of points on the WSB selected according to the criteria described in (7). The black points are points $w_{i}^{* *}$ that are within $\delta$ from the corresponding points $w_{i}^{*}$; these are type $A$ points. From left to right: (a) $n=1$ and $e=0.4$, (b) $n=2$ and $e=0.4$.

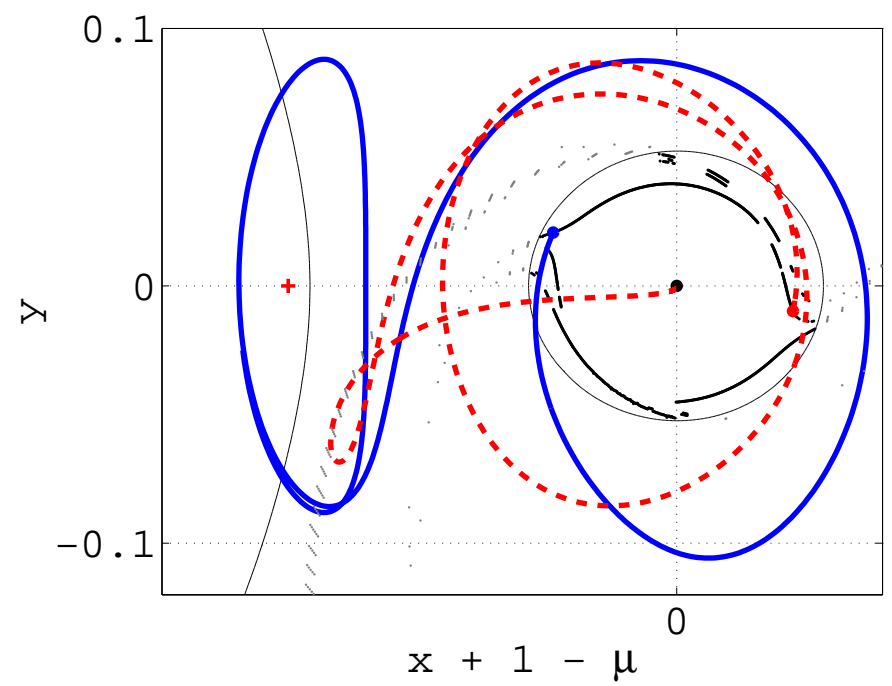

FIG. 4.7. Trajectory of a type A point lying on the stable manifold of a Lyapunov orbit (solid). Trajectory of a type $B$ point undergoing a close encounter with the primary (dashed).

symbolic dynamics. In Fig 4.7 a type A point and a type B point of the WSB are chosen inside the region bounded by the energy curve. The trajectory of the type A point approaches asymptotically a Lyapunov orbit for the corresponding energy level, therefore the type A point lies on the stable manifold of the Lyapunov orbit. Meanwhile the trajectory of the type B point undergoes a close encounter with the primary.

In Fig. 4.8 we show the trajectories of a collection of type A points chosen from the WSB. These trajectories approach asymptotically the Lyapunov orbit about $L_{1}$ or the Lyapunov orbit about $L_{2}$ corresponding to the energy level. Therefore these points lie on the union of the stable manifolds of the Lyapunov orbits about $L_{1}$ and $L_{2}$. 

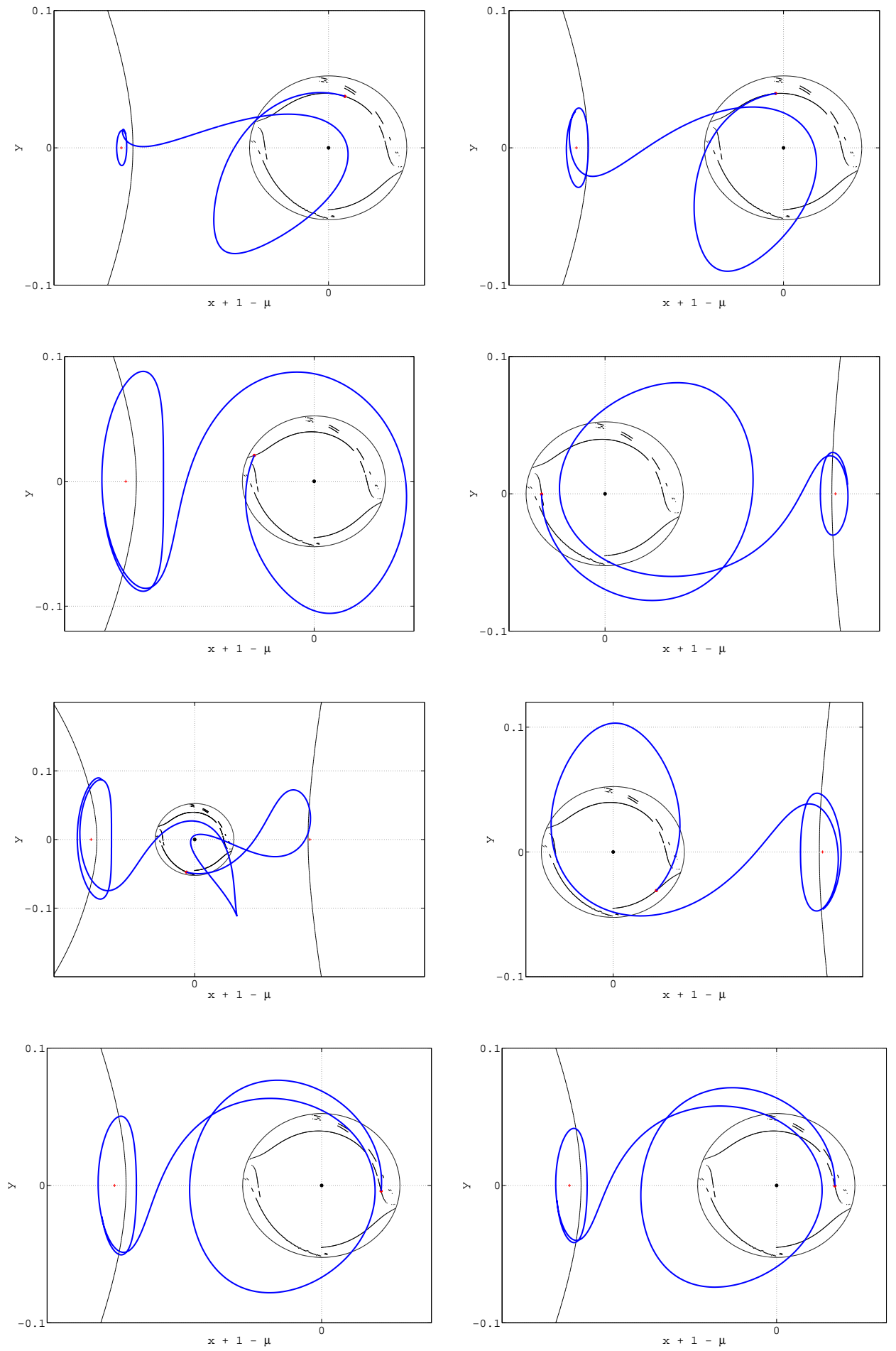

FIG. 4.8. Collection of trajectories of type A points lying on the stable manifolds of Lyapunov orbits for the corresponding energies. 

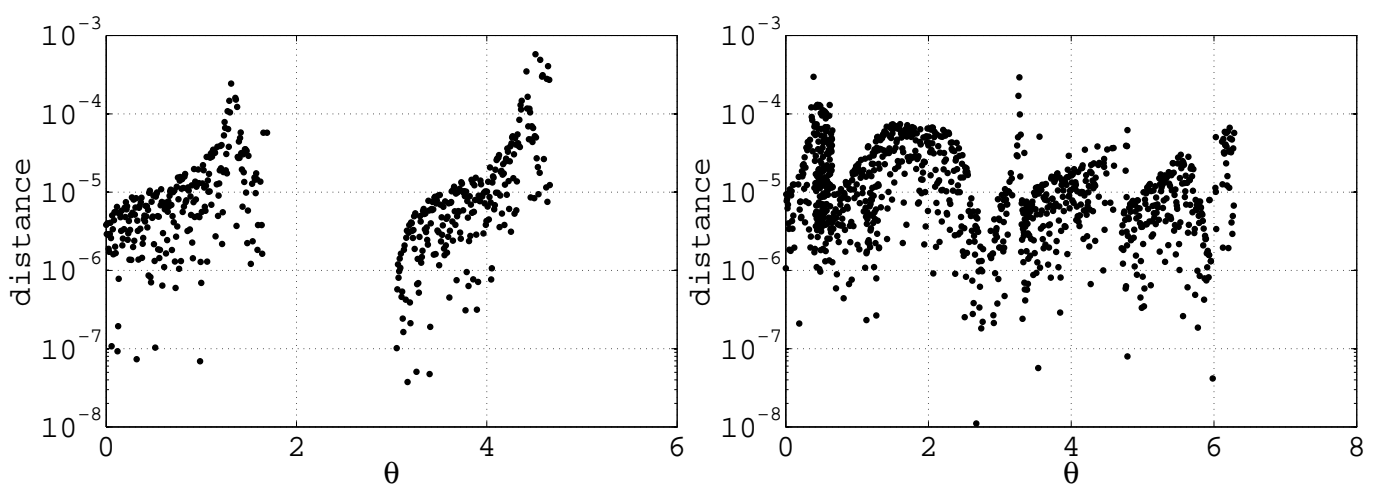

FIG. 4.9. Distribution of errors between the weak stability points and the points on the stable manifolds that satisfy the zero radial velocity condition and the negative Kepler energy condition relative to $P_{2}$. The horizontal axis represents the angle coordinate $\theta$ about $P_{2}$ and the vertical axis represents the distance between the points. From left to right: (a) $n=1$ and $e=0.4$, (b) $n=2$ and $e=0.4$.

In Fig. 4.9 we show the distribution of errors $d=\left\|w_{i}^{*}-w_{i}^{* *}\right\|$ for the type $\mathrm{A}$ points, for $C=\left[C_{\min }, C_{2}\right], n=1,2$ and $e=0.4$. The horizontal axis represents the angle coordinate $\theta$ about $P_{2}$ while the vertical axis shows the distance between the original point $w_{i}^{*}$ from $\mathcal{W}_{n}^{*}(e)$ and the matching point at the corresponding intersection between $W^{s}\left(\gamma_{1}\right)$ or $W^{s}\left(\gamma_{2}\right)$ and the axis $\dot{r}=0$ in $\mathcal{S}_{\theta_{i}^{*}}$. Most of these selected points meet a tolerance level of $10^{-4}$, while a few of them only meet a tolerance level of $10^{-3}$.

EXAMPLE 4.7. In WSB there are regions where the points of $W^{s}\left(\gamma_{1}\right)$ are intertwined with those of $W^{s}\left(\gamma_{2}\right)$. See Fig. 4.10. Since the points in $W^{s}\left(\gamma_{1}\right)$ and $W^{s}\left(\gamma_{2}\right)$ cannot overlap, we want to examine this region closer. For example, we choose a point $w_{i}^{*} \in \mathcal{W}_{1}^{*}(0)$ of Jacobi constant $C_{i}=3.159347461$. We compute the stable manifolds $W^{s}\left(\gamma_{1}\right)$ and $W^{s}\left(\gamma_{2}\right)$ and we intersect them with the corresponding Poincaré section $\mathcal{S}_{\theta_{i}^{*}}$. In this section, the cut made by $W^{s}\left(\gamma_{2}\right)$ appears to be a topological circle and the cut made by $W^{s}\left(\gamma_{1}\right)$ appears to be a broken circle. Both circles intersect the horizontal axis $\dot{r}=0$. Zooming in, although both intersections are close to the original point $w_{i}^{*}$ on the WSB, it turns out that it is the intersection point $w_{i}^{1}$ of $W^{s}\left(\gamma_{1}\right)$ with $\dot{r}=0$ that is closer. See Fig. 4.11. Of course $W^{s}\left(\gamma_{2}\right)$ also has an intersection point $w_{i}^{2}$ with $\dot{r}=0$ in the section, but this point does not correspond to $e=0$, so it actually belongs to a different $\mathrm{WSB}$ set $\mathcal{W}_{1}^{*}(e)$ with $e \neq 0$ small. When we take both intersection points $w_{i}^{1}, w_{i}^{2}$ and integrate them forward, the point $w_{i}^{1}$, which is essentially the same as the original point on the WSB, when flown forward, generates an orbit that gets close to $\gamma_{2}$ (because this point is close to $W_{\gamma_{2}}^{s}$ ), but then it changes course and approaches asymptotically $\gamma_{1}$ (because it is on $W_{\gamma_{1}}^{\gamma_{2}}$ ). See Fig. 4.10. This orbit is close to a heteroclinic connection between $\gamma_{2}$ and $\gamma_{1}$. For comparison, we also integrate forward the second point $w_{i}^{2}$, which is also close to the original point $w_{i}^{*}$ on the WSB. This second orbit is asymptotic to $\gamma_{2}$. The orbits of $w_{i}^{1}$ and of $w_{i}^{2}$ are very close to one another and seem to overlap for a while, but their final behavior is rather different. Note that the plotted orbits do not remain close to $\gamma_{1}$ and $\gamma_{2}$, respectively, as theoretically predicted, due to the numerical manifolds computed.

EXAMPLE 4.8. An important remark is that the points in the WSB set $\mathcal{W}_{n}^{*}(e)$ in general lie on different Jacobi energy levels. Indeed, for a fixed value of the eccentricity 


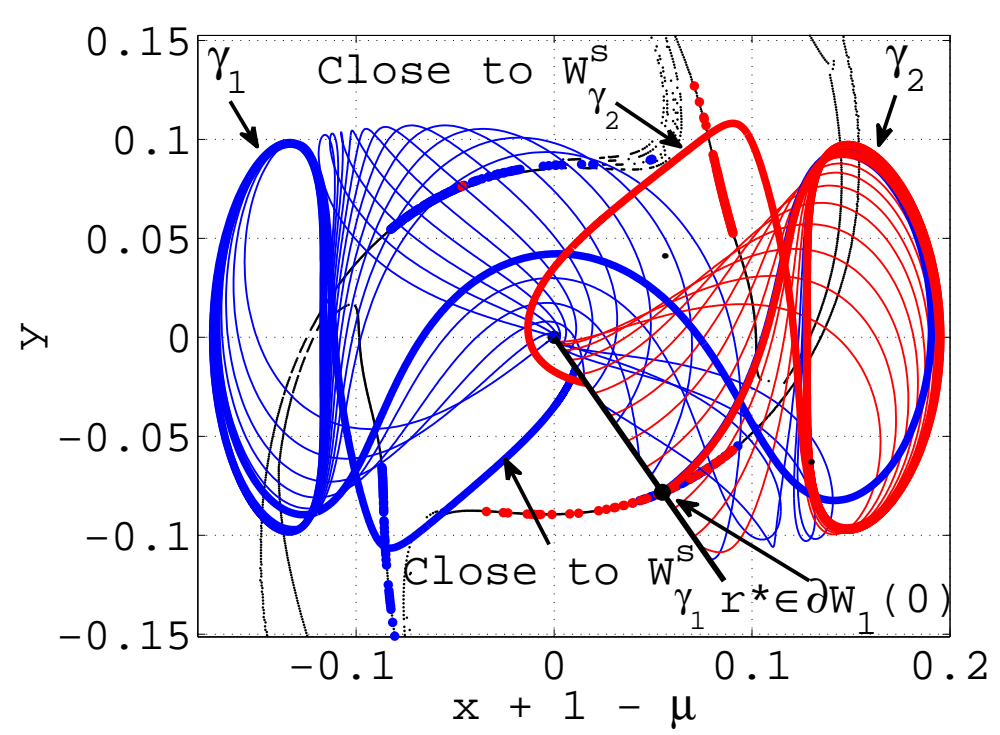

FIG. 4.10. The blue points in the WSB correspond to $W^{s}\left(\gamma_{1}\right)$, and the red points correspond to $W^{s}\left(\gamma_{2}\right)$. Forward integration of two initial points in the intersections of $W^{s}\left(\gamma_{1}\right)$ and $W^{s}\left(\gamma_{2}\right)$ with $\dot{r}=0$.
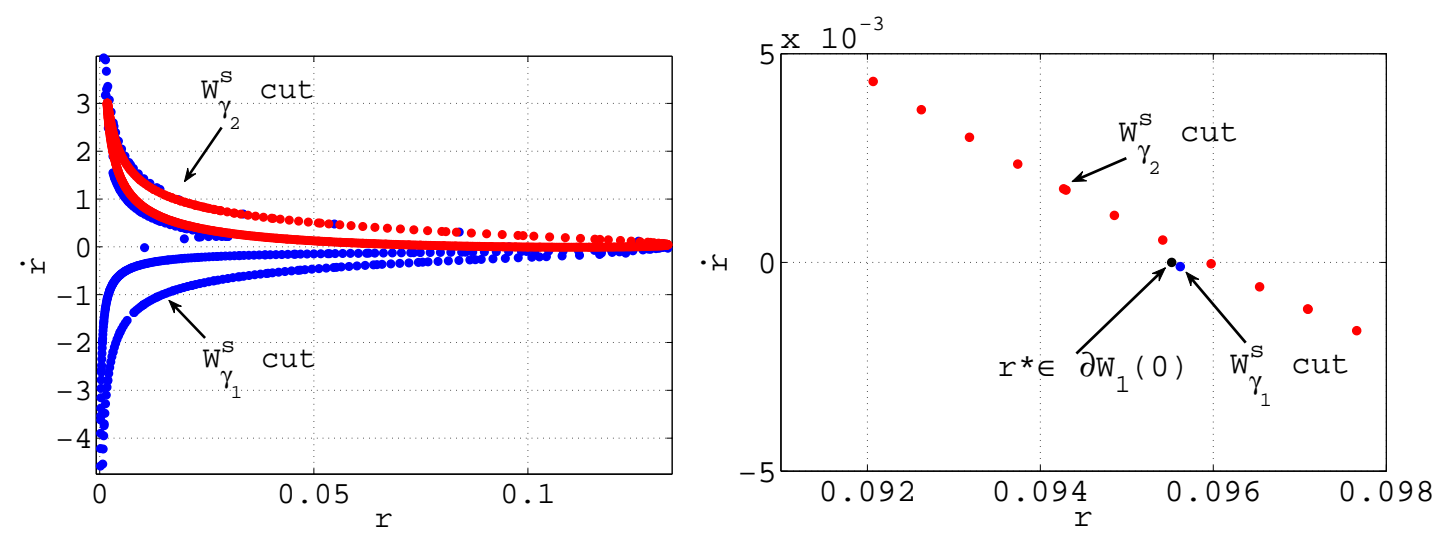

FIG. 4.11. Left: the cuts made by $W^{s}\left(\gamma_{1}\right)$ and $W^{s}\left(\gamma_{2}\right)$ to a Poincaré section. Right: identification of a point in the WSB.

$e$ and a fixed radial axis $l(\theta)$, a point $w^{*} \in \mathcal{W}_{n}^{*}(e)$ corresponds to a precise Jacobi energy level $J\left(r^{*}, \theta, e\right)$ given by $(2.7)$. Thus, by moving along the radial axis $l(\theta), J$ changes according to (2.7). However, only certain points on the radial axis $l(\theta)$ will be in the WSB set $\mathcal{W}_{n}^{*}(e)$. When the Jacobi constant corresponding to $\mathcal{W}_{1}^{*}(0)$ is plotted versus the angle $\theta$ one obtain the structure shown in Fig. 4.12. The two dips in the plot correspond to the two spiral arms of the set $\mathcal{W}_{1}^{*}(0)$. We note that the points on the two spiral arms reach very low values of the Jacobi energy, corresponding to the case when the Hill's region allows a trajectory to move within the entire plane.

4.3. Summary of geometrical and numerical arguments. We provided a geometrical argument that for a range of energy $C \in\left[C_{\min }, C_{1}\right], C_{\min }=3.15$, the 

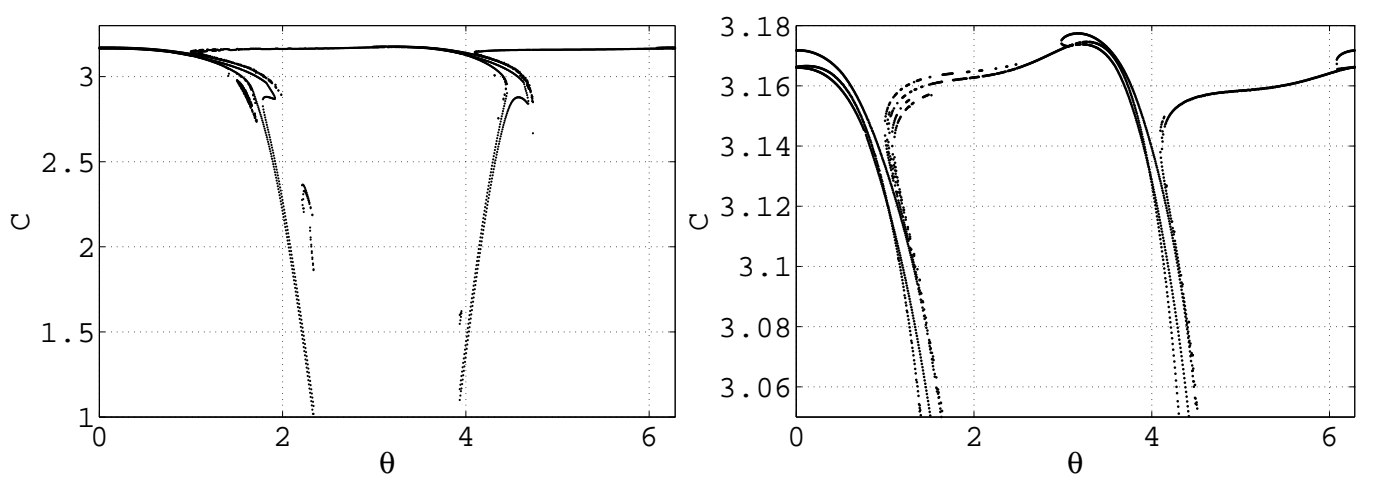

FIG. 4.12. The distribution Jacobi constants corresponding to $\mathcal{W}_{1}^{*}(0)$ plotted against the angle $\theta$ about $P_{2}$.

points on $W^{s}\left(\gamma_{1}\right) \cup W^{s}\left(\gamma_{2}\right)$ that have zero radial velocity and negative Kepler energy relative to $P_{2}$ are part of the WSB set, provided that the invariant manifolds of $\gamma_{1}$ and $\gamma_{2}$ satisfy some topological conditions described in Hypothesis A. The geometrical argument relies on the separatrix property of the invariant manifolds. We considered the cuts $W_{\theta_{0}, n-1}^{s}\left(\gamma_{1}\right)$ and $W_{\theta_{0}, n-1}^{s}\left(\gamma_{2}\right)$ made by $W^{s}\left(\gamma_{1}\right)$ and $W^{s}\left(\gamma_{2}\right)$ with $\mathcal{S}_{\theta_{0}}$. If a point is outside the region bounded by $W_{\theta_{0}, n-1}^{s}\left(\gamma_{1}\right)$ or $W_{\theta_{0}, n-1}^{s}\left(\gamma_{2}\right)$ then it will stay in the $P_{2}$-region for at least $n$-turns around $P_{2}$. If a point is inside the region bounded by $W_{\theta_{0}, n-1}^{s}\left(\gamma_{1}\right)$ or $W_{\theta_{0}, n-1}^{s}\left(\gamma_{2}\right)$ then it will leave the $P_{2}$-region after $(n-1)$-turns around $P_{2}$ and then it will make a complete turn around $P_{1}$. Thus the points on $W_{\theta_{0}, n-1}^{s}\left(\gamma_{1}\right)$ and $W_{\theta_{0}, n-1}^{s}\left(\gamma_{2}\right)$ with $\dot{r}=0$ and $H_{2}<0$ are points in $\mathcal{W}_{n}^{*}$.

We verified numerically this geometric argument for a selection of points. We computed the WSB set for a fixed eccentricity $e$. For a given point in the WSB we computed $W^{s}\left(\gamma_{1}\right)$ and $W^{s}\left(\gamma_{2}\right)$, and their cuts $W_{\theta_{0}, n-1}^{s}\left(\gamma_{1}\right)$ and $W_{\theta_{0}, n-1}^{s}\left(\gamma_{2}\right)$ with $\mathcal{S}_{\theta_{0}}$. We intersected the cuts with $\dot{r}=0$ and with the $H_{2}<0$ set. We found that one of these intersection points coincides, within some tolerance, with the original point chosen from the WSB. We tested the point by taking two nearby initial conditions inside the plane of section $\theta=\theta_{0}$ with $\dot{r}=0$, one inside the region bounded by $W_{\theta_{0}, n-1}^{s}\left(\gamma_{1}\right)$ or $W_{\theta_{0}, n-1}^{s}\left(\gamma_{2}\right)$ and the other one outside that region. We found that one point is stable and the other is unstable in the sense of the algorithmic definition of the WSB.

5. Conclusions. In this paper we provided a geometric argument, based on the separatrix property of the invariant manifolds of the Lyapunov orbits, for the fact that, for some range of energies, the points on the stable manifold of the Lyapunov orbits about $L_{1}$ and $L_{2}$ are points in the WSB, provided these points satisfy the zero radial velocity condition and the negative Kepler energy condition relative to the small primary. We supported our geometric argument with numerical experiments. This geometric argument justifies the numerical findings in [22]. The results in this paper, corroborated with those in [22], establish that the WSB method for the design of fuel efficient spacecraft trajectories substantially overlaps with the invariant manifold method.

A consequence of our findings is that the algorithmic definition of the WSB provides a method of finding trajectories on the stable manifold of a Lyapunov orbit which does not require the a priori knowledge of the Lyapunov orbit. In particular, 
one can obtain symmetric homoclinic orbits. This type of approach contrasts with the traditional method for computing the stable manifold of a Lyapunov orbit, which first computes the Lyapunov orbit and then integrates nearby initial conditions in the direction of the stable eigenvectors. It is well known that long term integration of the invariant manifolds is prone to large numerical errors. It seems possible that the algorithmic definition of the WSB can be used as a method of verification and correction for long term integration of the invariant manifolds.

The existence of homoclinic points inside the WSB is consistent with the results shown analytically in [6] in the case of near parabolic motion. However our numerical experiments indicate that the WSB contains symmetric homoclinic points for the whole range of Jacobi constants for which the WSB is defined.

Weak stability boundaries exist even in models where the hyperbolic invariant manifolds are no longer well defined. It seems possible that the WSB may turn out to provide a good substitute for the hyperbolic invariant manifolds in such models. We believe that this idea could be exploited in space mission design and it should be explored further.

Acknowledgement. M. G. would like to thank Gerard Gómez for useful discussions. Part of this work has been done during M.G.'s visits to the Centre de Recerca Matemàtica, Barcelona, Spain, and to Politecnico di Milano, Milano, Italy, for whose hospitality he is very grateful. Research of M.G. was partially supported by NSF grant: DMS 0601016. E.B. was supported by NASA SMD/AISR grants: NNG06GG55G, NNX09AK81G. He would also like to thank the Politecnico di Milano for their hospitality during his visits.

\section{REFERENCES}

[1] Alessi, E.M., Gómez, G., Masdemont, J.J.: Leaving the Moon by the means of the invariant manifolds of libration point orbits. Communications in Nonlinear Science and Numerical Simulation, 14, 4153-4167 (2009)

[2] Belbruno, E.: Lunar capture orbits, a method for constructing EarthMoon trajectories and the lunar GAS mission. Proceedings of AIAA/DGLR/JSASS Inter. Propl. Conf. AIAA paper No. 87-1054 (1987)

[3] Belbruno, E., Miller, J.: A ballistic lunar capture trajectory for the Japanese spacecraft hiten. Jet Propulsion Laboratory, IOM 312/90.41371-EAB (1990)

[4] Belbruno, E. and Marsden, B.: Resonance Hopping in Comets. The Astronomical Journal, 113, 1433-1444 (1997)

[5] Belbruno, E. and Miller, J.: Sun-Perturbated Earth-to-Moon Transfers with Ballistic Capture. Journal of Guidance, Control and Dynamics, 16, 770-775 (1993)

[6] Belbruno E.: Capture Dynamics and Chaotic Motions in Celestial Mechanics. Princeton University Press, Princeton (2004)

[7] Belbruno E.: Fly Me to the Moon: An Insiders Guide to the New Science of Space Travel. Princeton University Press, Princeton (2007)

[8] Broucke R.A.: Periodic Orbits in the Restricted Three-Body Problem with. Earth-Moon Masses, NASA-JPL Technical Report 32-1168, (1968)

[9] Burns, K. and Gidea, M.: Differential Geometry and Topology: With a View to Dynamical Systems. Chapman \& Hall/CRC, Boca Raton FL (2005)

[10] Canalias, E. and Masdemont, J.J.: Homoclinic and Heteroclinic Transfer Trajectories Between Planar Lyapunov Orbits in the Sun-Earth and Earth-Moon Systems. Discrete And Continuous Dynamical Systems - Series A, 14, 261-279 (2006)

[11] Celletti, A. and Chierchia, L.: KAM stability and celestial mechanics. Mem. Amer. Math. Soc. 187 (2007)

[12] Circi, C., Graziani, F., Porfilio, M., Teofilatto, P.: Weak Stability Boundary lunar transfers from Soyouz and Rockot planar launches. In: Proceedings of the 22nd International Symposium on Space Technology and Science, Paper No. ISTS 2000-j-01, 1555-1563, Morioka (2000) 
[13] Circi, C., Teofilato, P.: On the dynamics of weak stability boundary lunar transfers. Celestial Mech. Dyn. Astron. 79, 41-72 (2001)

[14] Circi, C., Teofilato, P.: Weak stability boundary trajectories for the deployment of lunar spacecraft constellations. Celestial Mech. Dyn. Astron. 95, 371-390 (2006)

[15] Conley, C.: Low Energy Transit Orbits in the Restricted Three-Body Problem. SIAM Journal of Applied Mathematics 16, 732-746 (1968)

[16] Doedel, E.J., Romanov, V.A., Paffenroth, R. C., Keller, H. B., Dichmann, D. J., Galán-Vioque, J., Vanderbauwhede, A.: Elemental Periodic orbits Associated with the libration Points in the Circular Restricted 3-Body Problem. International Journal of Bifurcation and Chaos 17, 2625-2677 (2007)

[17] Easton, R. W. and McGehee, R.: Homoclinic phenomena for orbits doubly asymptotic to an invariant three-sphere. Indiana Univ. Math. J. 28, 211-240 (1979)

[18] Gomez, G., Llibre J., Martinez R., Simo, C., : Dynamics and Mission Design Near Libration Points, Volume I : Fundamentals: The Case of Collinear Libration Points. World Scientific Monograph Series in Mathematics, (2001)

[19] Gomez, G., Llibre J., Martinez R., Simo, C., : Dynamics and Mission Design Near Libration Points, Volume II : Fundamentals: The Case of Triangular Libration Points. World Scientific Monograph Series in Mathematics, (2001)

[20] Gómez, G., Jorba, Á., Simó, C., and Masdemont, J.J.: Dynamics and Mission Design Near Libration Points Volume III: Advanced Methods For Collinear Points. World Scientific Monograph Series in Mathematics, (2001)

[21] Gómez, G., Jorba, Á., Simó, C., and Masdemont, J.J.: Dynamics and Mission Design Near Libration Points, Vol. IV: Advanced Methods for Triangular Points. World Scientific Monograph Series in Mathematics, (2001)

[22] García, F. and Gómez, G.: A Note on Weak Stability Boundaries. Celestial Mechanics and Dynamical Astronomy 97, 87-100 (2007)

[23] Gawlik, E. S., P. C. Du Toit, S. Campagnola and J. E. Marsden: Lagrangian coherent structures in the planar elliptic restricted three-body problem. Celest. Mech. Dyn. Astr 103, 227-249, (2009).

[24] Gidea M. and Masdemont J.J.: Geometry of Homoclinic Connections in a Planar Circular Restricted Three-Body Problem. International Journal of Bifurcation and Chaos 17 11511169 (2007)

[25] Jehn, R., Campagnola, S., García, D., \& Kemble, S.: Low-thrust approach and gravitational capture at Mercury, Proc. of the $18^{\text {th }}$ Int. Symp. on Space Flight Dynamics, 487-492, (2004).

[26] Jerg, S., Junge, O., Ross, S.: Optimal capture trajectories using multiple gravity assist. Communications in Nonlinear Science and Numerical Simulation, 14, 4168-4175 (2009)

[27] Llibre, J., Martínez, R., and Simó, C.: Transversality of the Invariant Manifolds Associated to the Lyapunov Family of Periodic Orbits near $L_{2}$ in the Restricted Three-Body Problem. Journal of Differential Equations 58, 104-156 (1985)

[28] Lo, M. and S. Ross. SURFing the Solar System: Invariant Manifolds and the Dynamics of the Solar System. JPL IOM 312/97, 2-4 (1997)

[29] Lo, M. W., Anderson, R. L., Whiffen, G., and Romans, L.: The Role of Invariant Manifolds in Low Thrust Trajectory Design. Advances in the Astronautical Sciences (AAS 04-288) 119, 2971-2990 (2004)

[30] Koon, W.S., Lo, M.W., Marsden, J.E., and Ross, S.D.: Heteroclinic connections between periodic orbits and resonance transitions in celestial mechanics. Chaos 10, 427-469, (2000).

[31] Koon W.S.,Lo M.W., Marsden J.E., Ross S.D.: Low Energy Transfer to the Moon. Celestial Mechanics and Dynamical Astronomy 81 63-73 (2001)

[32] Kummer, M.: On the stability of Hill's solutions of the plane restricted three-body problem. Am. J. Math. 101, 1333-1354 (1979).

[33] Macau, E.E.N.: Book Review: Capture Dynamics and Chaotic Motions in Celestial Mechanics by Edward Belbruno, Princeton University Press, Princeton/Oxford, 2004. Celestial Mechanics and Dynamical Astronomy 91, 407-408 (2005)

[34] McGehee, R.P.: Some homoclinic orbits for the restricted three-body problem. Ph.D. Thesis, University of Wisconsin, 1969.

[35] de Melo, C.F. and Winter, O.C.: Alternative paths to Earth-Moon transfer. Mathematical Problems in Engineering 2006, 1-20 (2006)

[36] Moser, J.: Stable and Random Motions in Dynamical Systems. Princeton University Press, Princeton, NJ (1973)

[37] Poschel, J.: On Nekhoroshev's Estimate for quasi-convex Hamiltonians. Math. Z. 5, 187-216 (1993)

[38] Romagnoli, D. and Circi, C.: Earth Moon Weak Stability Boundaries in the restricted three 
and four body problem. Celestial Mechanics and Dynamical Astronomy, 103 79-103 (2009)

[39] Ross, S.: Fly Me to the Moon - A Book Review. Notices of the American Mathematical Society 55, 478-480 (2008)

[40] Schoenmaekers, J., Horas, D. and Pulido, J.A.: SMART-1: with Solar Electric Propulsion to the Moon. 16th International Symposium on Space Flight Dynamics, Pasadena, California, 3-7 December (2001)

[41] Siegel, C.L. and Moser, J.: Lectures on celestial mechanics. Volume 187 of Grundlehren Math. Wiss., Springer-Verlag, Berlin-Heidelberg (1971)

[42] Simó, C. and Stuchi, T.: Central stable/unstable manifolds and the destruction of KAM tori in the planar Hill problem. Physica D, 140, 132 (2000)

[43] Szebehely, V.: Theory of Orbits. Academic Press, Orlando (1967)

[44] Topputo, F. and Belbruno, E.: Computation of the Weak Stability Boundaries: Sun-Jupiter System. Celestial Mechanics and Dynamical Astronomy, vol. 105, no. 1, 3-17 (2009)

[45] Yagasaki, K.: Sun-perturbed Earth-to-Moon transfers with low energy and moderate flight time. Celestial Mech. Dynam. Astronom. 90, 197-212 (2004) 